Trasformare le norme. II case-study del sex trafficking minorile in Thailandia

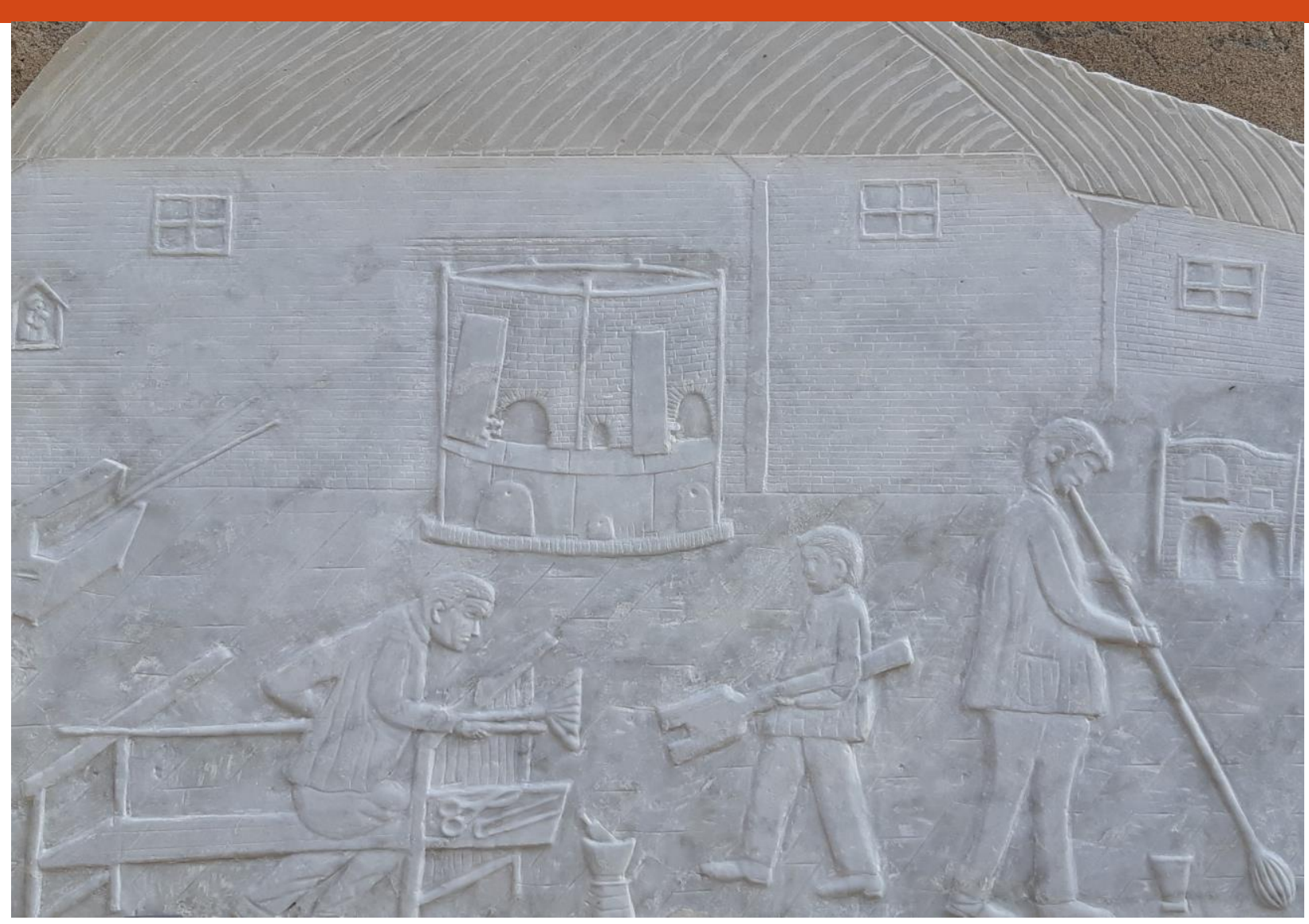

2021 ANNO VI NUMERO 12

di Riccardo Mazzola e Davide Rancati Dol https://doi.org/10.54103/2531$6710 / 17355$ 


\section{TRASFORMARE LE NORME. IL CASE-STUDY DEL SEX TRAFFICKING MINORILE IN THAILANDIA}

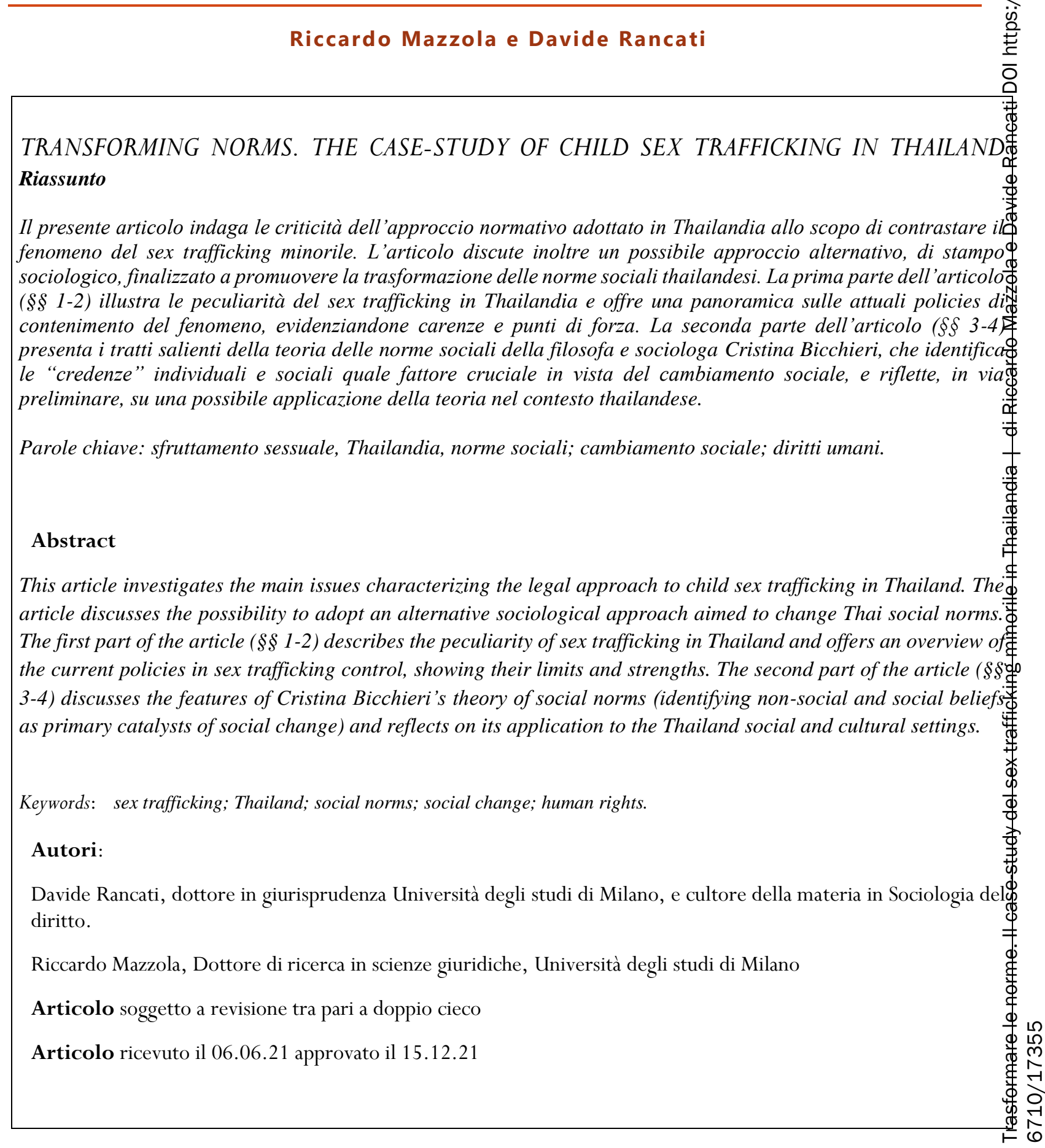




\section{Introduzione}

Agli inizi degli Anni '90, il mondo occidentale iniziò a conoscere le storie di prostituzione minorile provenienti dallo Stato thailandese. Grazie ad un massiccio monitoraggio da parte dei media europei e statunitensi, si portarono alla luce le vicende di sfruttamento sessuale di bambini che venivano venduti dai propri genitori, al prezzo di un elettrodomestico, per poi essere costretti ad offrire il proprio corpo ai desideri sessuali dei clienti dei bordelli cittadini (brothels). I dettagli di queste vicende non lasciavano spazio all'immaginazione: le bambine ancora vergini, quasi sempre con età inferiore ai quindici anni, erano il prodotto più richiesto. Il loro prezzo si aggirava intorno agli 8000 baht (l'equivalente di circa 200 euro) per il primo richiedente, che poteva godersi l'esclusiva più ricercata del momento. Da lì, il prezzo cominciava a scendere fino ad assestarsi a circa 200 baht (10 euro) a prestazione. Il tutto, andando incontro ad abusi e malattie diffuse: i reportage giornalistici del tempo davano atto di casi di bruciature di sigarette sul corpo, tagli autoinflitti, gonorrea e sifilide, e, in modo sempre più crescente, presenza del virus causa dell'AIDS. A ciò si aggiungevano le problematiche psicologiche, come la depressione, la consapevolezza dell'infanzia interrotta e il presagio che quei bambini, replicando l'unica realtà che avevano conosciuto per oltre metà della loro vita, sarebbero diventati loro stessi persecutori, una volta divenuti adulti.

Queste notizie ebbero l'effetto di portare l'attenzione della comunità internazionale sulle pratiche che venivano perpetrate all'interno dei confini thailandesi, facendo pressioni sul governo statale affinché prendesse contezza del fenomeno e predisponesse misure legislative adeguate a garantire il rispetto dei diritti fondamentali dei minori sanciti, fra gli altri, dalla Convenzione delle Nazioni Unite sui Diritti dell'Infanzia e dell'Adolescenza ${ }^{1}$, di cui la Thailandia è Stato Parte. La disciplina stabilita con questo strumento - che rappresenta la convenzione sui diritti umani più ratificata al mondo - oltre a tutelare il diritto alla vita e al corretto sviluppo del bambino, mira a proteggere le specifiche necessità nonché il rispetto del superiore interesse del minore. Ciò ha comportato che venisse messa al bando ogni forma di abuso, sfruttamento e

\footnotetext{
${ }^{1}$ Adottata dall'Assemblea Generale delle Nazioni Unite con risoluzione n. 44/25 del 20 novembre 1989. Entrata in vigore il 2 settembre 1990. Stati Parti al 20 aprile 2021: 196.
} 
atto di crudeltà nei confronti di chi ha una età inferiore ai diciotto anni: su questo, gli articoli 34 e 35 sono risolutivi nell'individuare le obbligazioni in capo ai singoli Paesi:

Gli Stati parti si impegnano a proteggere il fanciullo contro ogni forma di sfruttamento sessuale e di violenza sessuale. A tal fine, gli Stati adottano in particolare ogni adeguata misura a livello nazionale, bilaterale e multilaterale per impedire:

1. che dei fanciulli siano incitati o costretti a dedicarsi a una attività sessuale illegale;

2. che dei fanciulli siano sfruttati a fini di prostituzione o di altre pratiche sessuali illegali;

3. che dei fanciulli siano sfruttati ai fini della produzione di spettacoli o di materiale a carattere pornografico.

Gli Stati parti adottano ogni adeguato provvedimento a livello nazionale, bilaterale e multilaterale per impedire il rapimento, la vendita o la tratta di fanciulli per qualunque fine e sotto qualsiasi forma.

La richiesta di applicazione della normativa a tutela del fanciullo, poi ripresa dalle misure sia internazionali sia locali contro la tratta di esseri umani - la cui dettagliata descrizione è affidata alla successiva sezione - ha spinto taluni studiosi ad indagare come questa normativa di prevenzione e repressione di ogni forma di sfruttamento nei confronti dei bambini possa trovare attuazione all'interno di un paradigma socio-culturale, quale pare essere quello thailandese, che, al contrario, incentiva tali pratiche.

Come evidenziato da Obokata (2006: 44), in quello specifico Stato del Sud-Est asiatico la pratica dello sfruttamento è stata costruita, prima di tutto, culturalmente. L'immissione di donne e bambine nel business della prostituzione per supportare le famiglie è stata tollerata ed esercitata per lungo tempo, come parte dei costumi e delle tradizioni thailandesi. Mentre alcuni genitori invitano i propri figli ad avviarsi a questa attività, in altri casi sono gli stessi minori a prendere la decisione di lasciare il paese di origine, animati dal desiderio di contribuire al supporto economico del proprio nucleo familiare. E ciò perché i valori culturali predominanti nel Paese creano nella comunità l'aspettativa che le ragazze - già in età preadolescenziale - si azionino per assistere finanziariamente la famiglia: a livello locale, questa è definita come la responsabilità delle figlie "di restituire i soldi del latte materno" (traduzione mia). 
Fra le ricerche più significative in materia, emerge quella dell'antropologa Heather Montgomery (2011) che, in base agli studi effettuati in loco, ha evidenziato come in Thailandia esistano almeno tre forme di prostituzione che interessano i minori: la prima, che riguarda le ragazze straniere che vengono trafficate a fini di sfruttamento sessuale dagli Stati limitrofi (come la Cina, la Birmania, il Laos, la Cambogia) mediante l'uso della forza o dell'inganno impiegati dalle organizzazioni criminali²; la seconda, che concerne le ragazze che si prostituiscono nei bordelli, per loro scelta o per scelta dei genitori, in quanto "schiave del debito" [debt-bonded]; e la terza, che comprende i minori che vendono il proprio corpo spontaneamente per contribuire al sostentamento della propria famiglia (freelance) (Montgomery 2011: 785; traduzione mia).

Con riguardo al primo nucleo di persone, Montgomery riconosce che sono tra le categorie più esposte, perché la mancanza di cittadinanza e la marginalizzazione sociale le pone in una condizione di assoluta vulnerabilità, sia quando sono sottoposte allo sfruttamento nei bordelli (spesso gestiti dalle c.d. mama-sans) sia quando vengono salvate dalle forze di polizia o dalle organizzazioni non governative ${ }^{3}$. Spesso, infatti, vengono incriminate per violazione di leggi statali (ad esempio, immigrazione illegale), piuttosto che essere tutelate come vittime di tratta. Questo, indubbiamente, apre il campo ad altri temi che vanno oltre il mero sfruttamento, quali l'ineguaglianza sociale, le politiche immigratorie, la corruzione della polizia e le discriminazioni razziali.

Le fanciulle di origine thailandese, invece, sono più frequentemente sottoposte alla pratica del debt bondage, che mette anch'esse a rischio di sfruttamento sessuale, ma il pericolo scaturisce principalmente o dai loro parenti o dagli intermediari con i trafficanti presenti nelle loro comunità. Nella maggior parte dei casi, invero, le ragazze, per decisione propria o incentivate dai genitori, iniziano a lavorare nei bordelli - talvolta inconsapevoli che quella sarà la destinazione ultima del loro viaggio - in cambio di un pagamento anticipato

\footnotetext{
2 Secondo quanto riportato dal Trafficking in Persons Report (di seguito anche "TIP Report 2020") redatto dallo U.S. Department of State nel giugno 2020, i minori migranti sono, perlopiù, vittime di sfruttamento sessuale in bordelli, saloni di massaggio, bar, sale karaoke, hotel e residenze private.

${ }^{3}$ Con il termine rescue industry si intende l'attività anti-traffico posta in essere da taluni membri delle organizzazioni non governative che operano in loco, consistente nel prelevare la vittima dai bordelli durante incursioni notturne per portarla in posti sicuri, senza il contributo delle autorità statali o delle agenzie internazionali che si occupano di tratta di persone. Questa tipologia di pratica è stata più volte contestata, perché attuata da associazioni che spesso si disinteressano del contesto e della situazione in cui operano, esponendo così il minore al rischio di una sua re-vittimizzazione (Jones et al. 2017: 2).
} 
a favore della famiglia, e poi continuano a lavorare lì fino a quando il debito non è considerato come integralmente pagato ${ }^{4}$.

Infine, nel terzo gruppo di minori rientrano coloro che scelgono volontariamente di prostituirsi perché ritengono che questa forma di lavoro sia la migliore fra quelle disponibili (rispetto, ad esempio, ai lavori in campo agricolo o manifatturiero). In questo caso, poi, la prostituzione rappresenterebbe la continuazione di antichi modelli culturali di dovere filiale (filial obligation). Se le precedenti generazioni si prendevano cura dei loro genitori vendendo cibo o altre forme di piccolo commercio, la generazione moderna compie i suoi doveri verso i parenti più anziani attraverso il lavoro sessuale: queste giovani donne rimangono figlie fedeli inviando il proprio salario alle loro famiglie e fungendone da perno finanziario. Una volta terminato il periodo di lavoro, il loro re-inserimento sociale nella comunità di origine dipende da quanto sono state virtuose nel ruolo di prostitute e - dunque - nel foraggiare le necessità consumistiche della famiglia: solo chi ha fallito in questo compito, o si è tenuta i soldi per se stessa, viene stigmatizzata dal gruppo, a dimostrazione che la prostituzione non è considerata di per sé una attività moralmente inaccettabile o intrinsecamente dannosa per le ragazze (Montgomery 2011: 789-790). Al contrario, sembra suggerire Montgomery, questa attività consente alle giovani donne di responsabilizzarsi e di assumere un ruolo "da adulte" nella società in cui sono inserite: l'applicazione tout court delle disposizioni della menzionata Convenzione del 1989 - che adotta una visione del bambino di matrice occidentale - potrebbe, invece, creare una rottura tra figli e genitori, distruggendo l'economia familiare e i consolidati equilibri della comunità locale 5 .

Prendendo a riferimento il contesto thailandese così come sopra descritto, il presente elaborato vuole fornire, allora, una riflessione su come sia recepita nel Paese l'esigenza internazionale di vietare la pratica del

\footnotetext{
${ }^{4}$ Gli studi etnografici di Montgomery (2011: 787-788) hanno rilevato come, sebbene le condizioni di taluni bordelli siano ancora estremamente severe, gli episodi di abusi estremi sono in diminuzione e, talvolta, alle ragazze viene riconosciuto il diritto di scegliere i propri clienti e le proprie condizioni di lavoro.

5 Contro questa lettura proposta da Montgomery, atta a sostenere l'importanza del relativismo culturale nei confronti della imposizione dei diritti universali presenti nelle convenzioni internazionali, si è schierato Turner (2006) quando ha sostenuto che il fondamento ontologico alla base della disciplina sui diritti umani consiste nel riconoscimento che l'essere umano è una creatura vulnerabile, da intendersi, in modo minimale ed elementare, come propensione alla debolezza e alla inevitabile mortalità. Proprio questa condizione permette all'uomo di sviluppare una reciproca empatia: ciò è possibile, però, solo a patto che questa situazione venga effettivamente riconosciuta. Nel caso della Thailandia, ammettere che esistono persone di giovane età che sono oggettivamente vulnerabili - nonostante la cultura del posto non li consideri già più come "bambini" - è un passaggio indispensabile per attuare misure di protezione da abusi e di assistenza nei loro confronti (Turner 2006: 53-54).
} 
sex trafficking minorile, implementando così la tutela dei diritti universalmente riconosciuti, rispetto ad un contesto che, in taluni casi, pare dare priorità ad valori sociali che stanno agli antipodi rispetto a tale necessità. Il secondo paragrafo avrà ad oggetto, da un lato, l'illustrazione della normativa internazionale anti-tratta, e, dall'altro, il recepimento della stessa nella legislazione thailandese, al fine di comprendere se e come lo Stato si sia adeguato allo standard di protezione dei diritti umani delle vittime di traffico, specialmente donne e bambini, richiesto dalla disciplina internazionale. L'inquadramento normativo sarà principalmente finalizzato a comprendere se le misure esistenti siano idonee a fronteggiare una pratica che, come visto, si fonda su un paradigma culturale radicato nel tempo, che spesso impedisce al minore di riconoscersi come vittima di traffico a fini sessuali. In questo senso, il terzo paragrafo prenderà a riferimento la teoria delle norme sociali, proposta dalla filosofa e sociologa Cristina Bicchieri, per esaminare i fattori che influenzano il prodursi di condotte collettive, con la finalità di identificare diverse strategie che possono indurre al cambiamento sociale. Strategie che saranno, poi, approfondite nel quarto ed ultimo paragrafo, dove si proporrà una applicazione della metodologia di Bicchieri alla casistica della prostituzione dei minori per "dovere filiale" come individuata da Montgomery, con la suggestione di proporre una innovazione del paradigma culturale in grado di massimizzare l'efficacia del già esistente approccio vittimo-centrico contro la tratta di persone.

\section{Trafficare esseri umani in Thailandia: profili di diritto internazionale e statale}

\subsection{La disciplina del Protocollo di Palermo}

Ai fini dell'analisi proposta da questo elaborato, è necessario comprendere preliminarmente che cosa si intenda per traffico (o tratta) di persone.

Se le origini del traffico di persone possono essere fatte risalire alle antiche pratiche della schiavitù e della tratta degli schiavi, tale attività ha acquisito un significato diverso dall'inizio del Ventesimo secolo: mentre, infatti, la schiavitù era comunemente associata al trasporto di persone dall'Africa verso l'Europa e Nord America, la tratta, inizialmente, venne intesa come un'attività di prostituzione e sfruttamento sessuale di donne e ragazze bianche all'interno del Vecchio Continente.

La smisurata crescita del business del traffico di esseri umani nei tempi moderni è stata, invece, alimentata da un'ampia varietà di fattori: (i) la fine della Guerra Fredda e la caduta della cortina di ferro, che hanno 
causato una impennata dei traffici dall'Europa dell'Est; (ii) le guerre provocate dai nuovi Stati indipendenti dell'Europa orientale, finalizzate a stabilire la loro struttura di governo, dove è mancata una efficace applicazione della legge; (iii) il processo di globalizzazione degli ultimi trent'anni, in particolare i progressi nelle comunicazioni e nei trasporti, che ha semplificato il perpetrarsi di tale crimine ad opera dei trafficanti 6 ; e, da ultimo, (iv) l'adozione di leggi e politiche restrittive sull'immigrazione da parte degli Stati più sviluppati (tutte destinazioni popolari per la tratta di persone trafficate), che si sono dimostrate controproducenti, dato che la mancanza di mezzi per la migrazione legale ha costretto le vittime ad utilizzare i servizi forniti dai trafficanti (Obokata 2006: 21).

Se queste sono le premesse, come ha efficacemente riassunto Roujanavong (2012), oggi il traffico di persone si presenta come un crimine odioso e diffuso presente in tutto il mondo e in quasi tutte le società. È considerato una forma di schiavitù moderna che causa sofferenza alle vittime, trattate allo stesso modo in cui lo erano gli schiavi in passato. La maggior parte degli illeciti legati al traffico di esseri umani sono transnazionali ed è gestita o controllata da gruppi criminali organizzati7. Questo crimine genera una tale quantità di denaro per i trafficanti che è al secondo posto dopo i crimini legati alla droga in termini di redditività̀ $^{8}$ La complessità, le caratteristiche internazionali e gli aspetti umani coinvolti nella tratta di esseri umani, più la mancanza di leggi efficienti, la scarsa comprensione di questo reato da parte delle forze dell'ordine, la potente influenza dei criminali organizzati e la debole cooperazione internazionale tra i Paesi interessati rendono la tratta di esseri umani un crimine a basso rischio ma ad alto profitto. Il traffico di persone, dunque, sfida la comunità mondiale a trovare soluzioni e pratiche efficaci e pratiche per combattere

\footnotetext{
${ }^{6}$ Sono in molti a ritenere che il traffico di esseri umani nelle sue forme attuali si sia creato all'interno del contesto del neoliberalismo economico esploso con l'avvento della globalizzazione degli anni ‘90. Fra il 1970 e il 1980, infatti, la tratta di persone era principalmente contenuta all'interno dei confini statali (tranne in alcune regioni del mondo, come il Sud-Est asiatico, dove erano già diffuse forme di sfruttamento oltre frontiera), ma l'accrescersi delle disparità economiche, la competitività dei mercati finanziari, la facilità negli spostamenti e la nascita del World Wide Web - tutti fattori che hanno spinto milioni di persone, inclusi donne e bambini, a migrare dalle zone di povertà in cerca di opportunità di lavoro - hanno trasformato il traffico di persone da fenomeno locale a forma di business di carattere globale nelle mani della criminalità organizzata transnazionale (Marshall 2001: 6-7). Per ulteriori approfondimenti sugli effetti socio-giuridici provocati dalla globalizzazione nei confronti della tratta di esseri umani, si veda Rancati (2019).

7In tal senso, è noto che un'ampia varietà di individui e gruppi prende parte alla tratta. Oltre alle organizzazioni criminali più influenti, quali gli Snakeheads (Cina), la Yakuฉa (Giappone), la mafia russa e quella italiana, spesso il crimine in esame viene perpetrato anche dai membri della famiglia, da amici e da figure di spicco delle comunità locali che attirano le potenziali vittime nel processo di traffico. Inoltre, ci sono prove che suggeriscono che anche i membri delle missioni di pace e di altre organizzazioni intergovernative abbiano partecipato al traffico di esseri umani (Obokata 2006: 2)

${ }^{8}$ L'industria legata al traffico di esseri umani ha accresciuto il proprio fatturato in modo esponenziale, passando dai 32 miliardi di dollari americani del 2006 ai 150 miliardi di dollari del 2018 (Riback 2018: 42).
} 
quello che può essere considerato come uno dei più gravi crimini contro l'umanità ad oggi perpetrati nel mondo (Roujanavong 2012: 1; traduzione mia) ${ }^{9}$.

La rapidità con cui si è espansa questa fattispecie ha costretto la comunità internazionale a rivedere gli strumenti legislativi con cui affrontarla: fino agli inizi degli anni 2000, invero, il solo strumento internazionale diretto a fronteggiare il fenomeno era la Convenzione delle Nazioni Unite per la soppressione del traffico di persone e dello sfruttamento della prostituzione altrui del $1949^{10}$ che mirava a proibire e controllare le pratiche connesse allo sfruttamento per fini sessuali. Codesto documento, tuttavia, non forniva una definizione di tratta (Gallagher 2001: 985), dando così “un quadro normativo datato e mal equipaggiato per perseguire i trafficanti e fornire un'adeguata protezione alle persone trafficate" (Chuang 2006; traduzione $\mathrm{mia})^{11}$.

La svolta, in tal senso, è avvenuta con l'adozione della Convenzione delle Nazioni Unite contro la criminalità organizzata transnazionale ${ }^{12}$ e dei due Protocolli Addizionali13, finalizzati, l'uno, a prevenire, reprimere e

\footnotetext{
9 Ai sensi dell'articolo 7, paragrafo 1, dello Statuto di Roma, entrato in vigore il $1^{\circ}$ luglio 2002 ed istitutivo della Corte Penale Internazionale con sede all'Aia, "per crimine contro l'umanità s'intende uno degli atti di seguito elencati, se commesso nell'ambito di un esteso o sistematico attacco contro popolazioni civili, e con la consapevolezza dell'attacco: a) omicidio; b) sterminio; c) riduzione in schiavitù; d) deportazione o trasferimento forzato della popolazione; e) imprigionamento o altre gravi forme di privazione della libertà personale in violazione di norme fondamentali di diritto internazionale; f) tortura; g) stupro, schiavitù sessuale, prostituzione forzata, gravidanza forzata, sterilizzazione forzata e altre forme di violenza sessuale di analoga gravità; h) persecuzione contro un gruppo o una collettività dotati di propria identità, inspirata da ragioni di ordine politico, razziale, nazionale, etnico, culturale, religioso o di genere sessuale, o da altre ragioni universalmente riconosciute come non permissibili ai sensi del diritto internazionale, collegate ad atti preveduti dalle disposizioni del presente paragrafo o a crimini di competenza della Corte; i) sparizione forzata delle persone; j) apartheid; $\mathrm{k}$ ) altri atti inumani di analogo carattere diretti a provocare intenzionalmente grandi sofferenze o gravi danni all'integrità fisica o alla salute fisica o mentale". Al successivo paragrafo 2 viene poi sancito che, agli effetti del paragrafo 1, "per «riduzione in schiavitù» s'intende l'esercizio su una persona di uno o dell'insieme dei poteri inerenti al diritto di proprietà, anche nel corso del traffico di persone, in particolare di donne e bambini a fini di sfruttamento sessuale".

10 Adottata dall'Assemblea Generale delle Nazioni Unite con risoluzione 317 (IV) del 2 dicembre 1949. Aperta alla firma a Lake Success - New York, il 21 marzo 1950. Entrata in vigore internazionale: 25 luglio 1951. Stati Parti al 20 Aprile 2021: 82.

${ }_{11}$ Ai sensi degli articoli 1 e 2 della Convenzione del 1949: "Le parti con la presente Convenzione convengono di punire qualsiasi persona che, per soddisfare le passioni altrui: 1) procura, adesca o rapisca al fine di avviare alla prostituzione un'altra persona anche se consenziente; 2) sfrutta la prostituzione di un'altra persona anche se consenziente. Le parti con la presente convenzione convengono ugualmente di punire qualsiasi persona che: 1) mantenga, diriga o amministri o contribuisca a finanziare una casa chiusa; 2) conceda o prenda in affitto, in tutto od in parte, un immobile o un altro luogo ai fini della prostituzione altrui".

12 Adottata dall'Assemblea Generale delle Nazioni Unite con risoluzione A/RES/55/25 del 15 novembre 2000. Entrata in vigore il 29 settembre 2003. Stati Parti al 20 aprile 2021: 190.

${ }_{13}$ Nonostante spesso si tenda a confondere le due fattispecie di trafficking e smuggling, anche perché la prima, talvolta, scaturisce proprio dalla seconda, è essenziale evidenziare che ci si trova innanzi a due pratiche distinte e con caratteristiche proprie. Le principali differenze tra contrabbando e traffico, infatti, sono il consenso e lo sfruttamento. In primo luogo, chi viene contrabbandato acconsente all'attività, anche se spesso esercitata in condizioni deplorevoli, mentre il traffico di persone non è mai consensuale. Si noti che quando le persone sono ingannate sulle condizioni, sul trasporto e sul debito, si rientra nella fattispecie di tratta di esseri umani. In secondo luogo, le vittime della tratta continuano ad essere sfruttate una volta raggiunto il paese di destinazione, mentre nel contrabbando la persona si separa dal trafficante nel punto di approdo. A ciò si aggiunga il fatto che il contrabbando si compone, necessariamente, di un movimento internazionale, mentre il traffico di persone può avvenire sia all'interno che all'esterno dei confini nazionali. Queste essenziali differenze si riflettono anche negli strumenti adottati a Palermo: l'uso della coercizione o dell'inganno da parte dei trafficanti e il successivo sfruttamento hanno l'effetto di etichettare le persone trafficate come vittime di abusi dei diritti umani, e questo rafforza la necessità di garantire la loro protezione anche quando varcano i confini di uno Stato e/o vi rimangono illegalmente. La definizione di contrabbando, invece, implica che le persone coinvolte partecipino volontariamente alla migrazione illegale e questo può fornire una giustificazione agli Stati per applicare misure rigorose come l'arresto, la detenzione e la deportazione contro di loro (Obokata 2006: 22).
} 
punire la tratta delle persone (trafficking in persons), in particolare donne e bambini ${ }^{14}$ e, l'altro, a combattere il contrabbando (smuggling) di migranti via terra, via mare e via aria ${ }^{15}$. Con specifico riguardo alla redazione del Protocollo per reprimere e punire la tratta di persone (di seguito anche il "Protocollo contro la Tratta" o il "Protocollo di Palermo"), a seguito di vivaci negoziazioni16, la definizione di traffico di persone adottata all'articolo 3 e, ad oggi, universalmente accettata (Riback 2018: 44), si compone di questi tre elementi17:

1. una azione che consiste nel reclutamento, trasporto, trasferimento, l'ospitare o accogliere persone;

2. attraverso l'impiego o la minaccia di impiego della forza o di altre forme di coercizione, di rapimento, frode, inganno, abuso di potere o di una posizione di vulnerabilità o tramite il dare o ricevere somme di denaro o vantaggi per ottenere il consenso di una persona che ha autorità su un'altra;

3. a scopo di sfruttamento, che comprende, come minimo, lo sfruttamento della prostituzione altrui o altre forme di sfruttamento sessuale, il lavoro forzato o prestazioni forzate, schiavitù o pratiche analoghe, l'asservimento o il prelievo di organi.

Affinché, dunque, si realizzi la descritta fattispecie, è necessario che tutti questi tre elementi siano integrati. La sola eccezione riguarda il caso in cui le vittime siano dei bambini, ossia qualsiasi persona al di sotto dei diciotto anni di età: in questa circostanza, il reclutamento, trasporto, trasferimento, l'ospitare o accogliere un bambino ai fini di sfruttamento si configurano come "tratta di persone" anche se non comportano l'utilizzo di nessuno dei mezzi sopra indicati.

\footnotetext{
${ }^{14}$ Adottato dall'Assemblea Generale delle Nazioni Unite con risoluzione A/RES/55/25 del 15 novembre 2000. Entrato in vigor il 25 dicembre 2003. Stati Parti al 20 aprile 2021: 178.

${ }^{15}$ Adottato dall'Assemblea Generale delle Nazioni Unite con risoluzione A/RES/55/25 del 15 novembre 2000. Entrato in vigore il 28 gennaio 2004. Stati Parti al 20 aprile 2021: 150.

${ }^{16}$ Riportando quanto sottolineato da Quiroz Vitale: “Sui lavori preliminari emergeva dunque un terreno di scontro su temi propri della modernità: a quali condizioni l'essere umano è in grado di esercitare una libera scelta? Una situazione di obiettiva vulnerabilità, riscattabilità, deficit cognitivo o intellettivo, un enorme gap culturale, la povertà estrema sono condizioni che possono influire sull'espressione della libertà dell'attore sociale? Il processo di "vittimizzazione" delle donne è una costruzione sociale, una etichetta che coarta la volontà delle donne o un processo sociale di riconoscimento del dovere di protezione nei confronti di soggetti i cui diritti umani sono violati? Quale rilievo morale può avere il consenso dello sfruttato o dello schiavo e quale incidenza può avere sull'azione degli Stati” (Quiroz Vitale 2018: 133).

${ }_{17}$ Per completezza, l'articolo 3 viene qui di seguito riportato integralmente: “Ai fini del presente Protocollo: a) «tratta di persone» indica il reclutamento, trasporto, trasferimento, l'ospitare o accogliere persone, tramite l'impiego o la minaccia di impiego della forza o di altre forme di coercizione, di rapimento, frode, inganno, abuso di potere o di una posizione di vulnerabilità o tramite il dare o ricevere somme di denaro o vantaggi per ottenere il consenso di una persona che ha autorità su un'altra a scopo di sfruttamento. Lo sfruttamento comprende, come minimo, lo sfruttamento della prostituzione altrui o altre forme di sfruttamento sessuale, il lavoro forzato o prestazioni forzate, schiavitù o pratiche analoghe, l'asservimento o il prelievo di organi; b) il consenso di una vittima della tratta di persone allo sfruttamento di cui alla lettera a) del presente articolo è irrilevante nei casi in cui qualsivoglia dei mezzi usati di cui alla lettera a) è stato utilizzato; c) il reclutamento, trasporto, trasferimento, l'ospitare o accogliere un bambino ai fini di sfruttamento sono considerati «tratta di persone» anche se non comportano l'utilizzo di nessuno dei mezzi di cui alla lettera a) del presente articolo; d) «bambino» indica qualsiasi persona al di sotto di diciotto anni”.
} 
Proprio questa previsione ci fornisce lo spunto per rimarcare l'imponente sforzo compiuto dal Protocollo in esame per disciplinare quella che è, tutt'oggi, una delle forme di traffico più diffuse al mondo, ossia lo sfruttamento nei confronti dei minori ${ }^{18}$. Già durante i lavori preparatori, infatti, le principali istituzioni internazionali a tutela dei diritti umani dei bambini sollecitarono il Comitato Ad Hoc a predisporre misure adeguate per contrastare questo fenomeno, sempre più diffuso a livello globale: dal tono della raccomandazione congiunta, emerse la necessità del primato del diritto internazionale dei bambini nei confronti delle pratiche nazionali:

[i]l Protocollo dovrebbe includere un esplicito riconoscimento del fatto che i bambini hanno diritti speciali in base al diritto internazionale, e in particolare alla luce della Convenzione sui diritti dell'infanzia; che i bambini vittime della tratta hanno bisogni speciali che devono essere riconosciuti e soddisfatti dagli Stati parti; che gli Stati sono obbligati ad adottare misure per prevenire il traffico di bambini; e che nel trattare con i bambini vittime della tratta, l'interesse superiore del bambino (compreso il diritto specifico al recupero fisico e psicologico e all'integrazione sociale) devono essere sempre al primo posto. È anche importante un chiaro riconoscimento della necessità di combattere l'impunità dei responsabili del traffico, assicurando allo stesso tempo che il bambino non sia criminalizzato in alcun modo. In questo contesto, va notato che la maggioranza degli Stati sono già soggetti a tali obblighi legali attraverso la loro ratifica della Convenzione sui diritti del bambino. Il diritto internazionale esistente richiede agli Stati di assicurare, tra l'altro, che l'assistenza e la protezione dei bambini vittime della tratta non siano rese discrezionali o comunque dipendenti dalla decisione delle autorità nazionali. In conformità con l'articolo 2 della Convenzione sui diritti dell'infanzia, i bambini vittime della tratta hanno diritto alla stessa protezione dei cittadini dello Stato di accoglienza in tutte le questioni, comprese quelle relative la protezione della loro vita privata e della loro integrità fisica e morale ${ }^{19}$.

Se, come sostenuto da Gallagher (2001: 989), la versione finale del Protocollo di Palermo è stata ben al di sotto di questo standard, quest'ultima contiene comunque una serie di previsioni finalizzate a garantire una

\footnotetext{
${ }^{18}$ Sulla base dei dati riportati dal Global Estimates of Modern Slavery Report redatto dall'International Labour Office (ILO) nel 2017, un quarto delle vittime mondiali di traffico sono bambini. Il 18\% sono assoggettati a sfruttamento per lavori forzati, mentre il $21 \%$ è impiegato in attività connesse allo sfruttamento sessuale.

${ }^{19} \mathrm{Si}$ veda Comitato Ad Hoc per l'elaborazione della Convenzione contro la criminalità organizzata transnazionale, nota dell'Alto Commissario delle Nazioni Unite per i diritti umani, del Fondo delle Nazioni Unite per l'infanzia, l'Alto Commissariato delle Nazioni Unite per i rifugiati e l'Organizzazione internazionale per le migrazioni sui progetti di protocollo riguardanti il traffico di migranti e la tratta di persone, U.N. Doc. A/AC.254/27, paragrafo 6 (traduzione mia).
} 
più ampia protezione alle vittime al di sotto dei diciotto anni, a partire proprio dal fatto che, quando è coinvolto un bambino, la forza o la coercizione non sono più considerati requisiti essenziali per integrare il reato di tratta. Oltre a ciò, il Protocollo contro la Tratta prevede specifiche misure di tutela che devono essere implementate dagli Stati Parti e che devono prendere in considerazione le speciali esigenze dei fanciulli, offrendo una adeguata sistemazione, educazione e cura, nel miglior interesse del bambino.

Senza addentrarci ulteriormente nelle vicissitudini che hanno caratterizzato la formazione del principale strumento normativo anti-traffico, in chiusura di questo paragrafo è fondamentale rimarcare quello che è stato l'autentico elemento caratteristico fornito al Protocollo contro la Tratta, ossia la necessità di relazionarsi al traffico di esseri umani mediante un approccio olistico in grado di porre la protezione dei diritti umani al centro di ogni azione repressiva e preventiva. E ciò anche al fine di escludere ogni forma di responsabilità penale nei confronti delle vittime, troppo spesso perseguite perché illegali nel Paese o per le attività che sono costrette a compiere nel loro ruolo di persone trafficate (prostituzione, ad esempio, laddove è proibita). Non a caso, la c.d. strategia delle "tre P" (prosecution, protection and prevention) adottata a Palermo ha, come suo fulcro, proprio l'assistenza e la tutela delle vittime, ai cui è riservato un set di misure che gli Stati Parti sono chiamati a prendere in considerazione nei rispetti territori, anche con l'obiettivo di evitare una nuova vittimizzazione, specialmente delle donne e dei bambini, che, da sempre, sono i soggetti più esposti a vulnerabilità ${ }^{20}$.

In linea con quanto evidenziato da Wylie e McRedmond (2010), dunque, l'adozione del Protocollo di Palermo e la sua tripartitica definizione di tratta si sono posti come elementi essenziali per portare il dibattito sul traffico di persone verso una nuova direzione e per permettere che quest'ultimo venisse considerato, dopo anni di tragiche incertezze, come una grave violazione dei diritti umani - e non più solo come un fenomeno migratorio / criminale - da parte della comunità internazionale.

\footnotetext{
${ }^{20}$ Nel dettaglio, l'articolo 6, comma 3, del Protocollo di Palermo sancisce che: “[o]gni Stato Parte prende in considerazione l'attuazione di misure relative al recupero fisico, psicologico e sociale delle vittime di tratta delle persone e, nei casi opportuni, in collaborazione con le organizzazioni non governative, altre organizzazioni interessate e altri soggetti della società civile, il fornire: a) un alloggio adeguato; b) consulenza e informazioni, in particolare in relazione ai loro diritti riconosciuti dalla legge, in una lingua che le vittime di tratta delle persone comprendano; c) assistenza medica, psicologica e materiale; e d) opportunità di impiego, opportunità educative e di formazione. Ogni Stato Parte prende in considerazione, nell'applicare le disposizioni del presente articolo, l'età, il sesso, ed esigenze speciali delle vittime di tratta delle persone, in particolare le esigenze specifiche dei bambini, inclusi un alloggio, un'educazione e delle cure adeguati. Ogni Stato Parte cerca di assicurare l'incolumità fisica delle vittime di tratta delle persone mentre sono sul proprio territorio. Ogni Stato Parte assicura che il proprio sistema giuridico interno preveda misure che offrono alle vittime di tratta delle persone la possibilità di ottenere un risarcimento per il danno subito".
} 


\subsection{Le cause storico-culturali del traffico di persone in Thailandia}

2.2.1. È fatto notorio che, all'interno delle regioni facenti parte dell'Associazione delle Nazioni del Sud-Est asiatico $^{21}$ (Association of Southeast Asia Nations, di seguito anche "ASEAN"), la pratica del traffico di persone sia un grave crimine che colpisce i diritti umani, la dignità e l'integrità delle vittime, siano esse donne, uomini o bambini. Le ragioni per cui tale area del pianeta è così colpita dal traffico di esseri umani sono innumerevoli. Nella letteratura di settore si riscontrano cause di natura socio-politica (quali, ad esempio, gli anni di conflitto nella regione, gli alti livelli di corruzione e le transizioni dal sistema comunista che hanno lasciato molte persone in posizioni di estrema vulnerabilità); cause di natura geografica, poiché vi sono ampie aree di confine dalla giurisdizione incerta sotto il controllo delle organizzazioni criminali; nonché cause di origine culturale. Con particolare riferimento a quest'ultime, vari autori hanno rilevato come le tradizioni familiari (in particolar modo, il patriarcato) giochino un ruolo fondamentale nel perpetuare la tratta (Shelley 2010: 158).

Nel corso dell'ultimo decennio sono stati compiuti numerosi sforzi per contrastare tale fenomeno, sia attraverso l'istituzione di leggi regionali sia attraverso l'operato di enti competenti nella salvaguardia dei diritti umani; ma, nonostante questi tentativi, l'ASEAN non è stata in grado di arginare suddetta piaga sociale (Kranrattanasuit 2014:1-2). Secondo i dati forniti dal Global Slavery Index 2018, sono oltre 3,5 milioni le persone sottoposte a sfruttamento lavorativo o per finalità sessuali all'interno della regione. Nello specifico, i numeri forniti dal Global Trafficking in Persons Report 2020, redatto annualmente dallo United Nations Office on Drugs and Crime (di seguito anche "UNODC Report 2020"), fotografano una situazione dove circa la metà delle vittime riconosciute sono donne adulte, mentre i bambini rappresentano circa un terzo delle vittime totali22.

Questa premessa era doverosa per fornire un inquadramento preliminare della situazione ad oggi presente nei confini del Sud-Est asiatico, dove la Thailandia primeggia nella lista degli Stati più colpiti dal traffico di persone. Innanzitutto, quest'ultima si presenta come un Paese che 'produce' persone trafficate, da destinarsi

\footnotetext{
${ }^{21}$ L'ASEAN, fondata nel 1967, opera con lo scopo principale di promuovere la cooperazione e l'assistenza reciproca fra gli Stati Membri e per accelerare il progresso economico e la stabilità all'interno della regione. Ad oggi, gli Stati facenti parte di questa organizzazione internazionale sono: Filippine, Indonesia, Malaysia, Singapore, Thailandia, Brunei, Vietnam, Birmania, Laos, Cambogia. Papua Nuova Guinea e Timor Est sono stati ammessi con lo status di osservatori.

${ }^{22}$ La maggior parte di esse continua ad essere trafficata a scopi di sfruttamento sessuale (oltre il 60\%, rispetto al $30 \%$ impiegata nel lavoro forzato), a cui si affiancano anche le nuove forme di sfruttamento quali gli abusi sessuali finalizzati alla produzione di materiale pornografico o pedo-pornografico e la tratta di persone per matrimoni forzati.
} 
all'estero: ne sono un esempio le migliaia di cittadini thailandesi che annualmente approdano illegalmente in Giappone per essere impiegate nelle aziende agricole, nelle fabbriche, nei ristoranti, o nel business della prostituzione $^{23}$. La Thailandia è, poi, un luogo di transito, grazie alla sua posizione strategica: anche in questo caso, sono innumerevoli le persone di altre cittadinanze (ad esempio, cinesi) che vengono trafficate attraverso i confini thailandesi per essere destinate alla Malesia, al Myanmar, al Vietnam. E infine, la Thailandia è un Paese utilizzato come meta finale da parte dei trafficanti, che letteralmente "importano" decine di migliaia di persone ogni anno dagli Stati limitrofi (quali la Cambogia, il Laos, il Myanmar) (Obokata 2006: 39-40).

2.2.2. Guardando alla storia del Paese, si può constatare che traffico di persone e la schiavitù abbiano radici talmente profonde che, spesso, ancora oggi l'attività del trafficante non viene stigmatizzata né considerata come deviante dalla comunità, facilitando così la sopravvivenza di tale pratica (Shelley 2010: 149). Ad esempio, nel Regno di Lanna, situato nel nord dell'attuale Thailandia dal XIII al XVIII secolo D.C., il re possedeva $\mathrm{i}$ terreni agricoli e se le persone non erano in grado di pagare le relative tasse (o erano in una qualunque altra posizione di debito nei confronti del monarca), potevano scegliere di vincolare sé stessi, le loro moglie, i loro figli o parenti minori alla c.d. "schiavitù del debito" (debt bondage). Gli esseri umani assoggettati a tale forma di schiavitù, nonostante la possibilità di essere liberati da questo obbligo, raramente riuscivano a riscattare il saldo, finendo invece per essere rivenduti a nuovi padroni. In questo senso, è emblematica la sorte a cui erano destinate le figlie dell'indebitato, che venivano impiegate all'interno del sistema di lavoro forzato denominato corvée $e^{24}$ per essere utilizzate come schiave domestiche e sessuali. Oltre a ciò, vi sono evidenze storiche che testimoniano come agli inizi del XIV secolo i governanti della Thailandia (allora chiamata Siam), autorizzarono e tassarono la prostituzione, intervenendo attivamente nella regolazione di tale lavoro (Rande Taylor 2003).

23 Oltre al Giappone, fra le destinazioni principali vi sono la Germania, gli Stati Uniti, il Regno Unito, il Sud-Africa, e l'Australia (Roujanavong 2012: 138).

${ }^{24}$ In termini generali, per corvée si intende la prestazione d'opera dovuta dai coltivatori che risiedevano su terreni dati in concessione (mansi) sotto forma di giornate di lavoro obbligatorie a favore del signore, proprietario dell'area. 
Come sottolineato da Davy (2014: 802), nonostante queste pratiche siano state formalmente abolite da Re Rama V nel 1095, le gerarchie sociali, le varie forme di schiavitù e lo sfruttamento dei figli per pagare i debiti familiari sono pratiche che hanno continuato a persistere nella Thailandia moderna.

Con specifico riguardo all'industria sessuale, questa ricevette una significativa accelerazione durante la ventennale Guerra del Vietnam, combattuta tra il 1955 e il 1975, quando oltre 40.000 membri dell'apparato militare statunitense entrarono nello Stato thailandese per trascorrervi periodi di riposo e svago dal conflitto armato in corso nella limitrofa regione. Al termine della guerra, fu l'espandersi della globalizzazione mondiale a segnare il nuovo trend per il Paese: da un lato, infatti, sempre più stranieri, soprattutto occidentali, cominciarono a varcare i confini thailandesi alla ricerca di facili e convenienti attività sessuali; dall'altro, invece, l'estrema povertà in cui versavano le comunità rurali del Paese e i conflitti armati in corso negli Stati confinanti (Laos, Cambogia, Birmania) costrinsero una sempre più alta quantità di minori a trasferirsi nei centri urbanizzati della Thailandia, dove - spinti dalla necessità di fuggire dall'indigenza vennero attirati nella rete dello sfruttamento sessuale, in continua espansione. Durante questo periodo di rapida crescita dell'industria del sesso, le azioni di contrasto intraprese dalle autorità thailandesi furono minime poiché, al contrario, fino al termine degli Anni '80 il governo promosse apertamente lo sviluppo di detto business, anche mediante leggi fiscali ad hoc mirate a tassare le donne coinvolte nella prostituzione (Hitchcock: 2008) ${ }^{25}$. Negli anni successivi, l'abrogazione formale di queste previsioni tributarie è stata sostituita dalla corruzione dei funzionari amministrativi che, in cambio di denaro, hanno tollerato che gli affari legati allo sfruttamento della prostituzione continuassero pressoché impuniti (Kranrattanasuit 2014: 105).

2.2.3. Se il profilo storico aiuta ad individuare le cause che hanno consentito alla tratta odierna di sorgere quasi incontrastata, è la prospettiva socio-culturale che ci permette di cogliere le sfumature più complesse ed articolate del fenomeno. Ciò che, invero, ha contribuito alla fioritura massiccia dello sfruttamento di certe categorie di individui, è la condizione speciale in cui si trovano le donne all'interno della società thailandese. Secondo le analisi condotte da Mensendiek (1997), il nucleo familiare si struttura intorno ai membri di sesso

\footnotetext{
25 Il riferimento è al Contagious Disease Act del 1908, che autorizzava l'attività di prostituzione nei bordelli a condizione che le prostitute pagassero le relative imposte allo Stato. Nonostante questo tentativo di monitoraggio, l'espansione della prostituzione nel Paese crebbe in modo incontrollato, perché un alto numero di donne non dichiarava la propria attività alle autorità competenti (Obokata 2006: 48).
} 
femminile e anche l'autorità di comandare, nonostante resti di stampo patriarcale, si trasmette attraverso la linea materna. Proprio questo ruolo principale all'interno della famiglia ha costretto la donna a cercare nuove forme di sostentamento della stessa per fronteggiare i cambiamenti derivanti dalla modifica del sistemo economico rurale. Infatti, il fallimento dell'economia basata principalmente sul lavoro agricolo ha forzato innumerevoli donne di ogni età a migrare, talvolta anche per anni, verso i poli urbanizzati - su tutti, la capitale Bangkok - con l'obiettivo di trovare e fornire un valido supporto di aiuto finanziario alle proprie famiglie: una volta ottenuti i soldi, invero, questi venivano usualmente inviati alle proprie case di origine come strumenti di mantenimento dell'intero gruppo familiare.

Ciò che rileva maggiormente di questo fatto sociale è che, come sottolineato da Davy (2014), le donne e le ragazze thailandesi si prestavano alla prostituzione e alla tratta sessuale non per impotenza, ma in virtù del senso di responsabilità che le legava alla famiglia, la quale, grazie al loro lavoro, otteneva una via di sopravvivenza rispetto alla povertà estrema in cui riversava. E ciò in linea anche con quanto avviene anche in altre regioni dell'Asia, dove il traffico di persone continua ad essere perpetrato perché c'è l'aspettativa che taluni membri della famiglia lavorino in condizioni degradanti o di sfruttamento per provvedere al benessere degli altri. La vendita di parte del nucleo familiare ai trafficanti viene fatta per liberare la famiglia di un elemento che non può essere sfamato o perché questo le fornisca il capitale necessario ai propri bisogni (Pink 2013: 170)

Questo sistema, perpetrato nel corso degli anni dopo l'avvento della globalizzazione, sussiste tutt'ora e trova la sua giustificazione anche nel ruolo decisivo giocato dalla discriminazione di genere, uno dei fattori chiave del traffico di persone in Thailandia. Le donne e le ragazze, infatti, in quanto sottostimate dall'oppressiva cultura patriarcale, hanno meno opportunità di ottenere lavori regolari, restando quindi più vulnerabili ed esposte alle mire dei trafficanti, che le adescano offrendo facili soluzioni di guadagno. Secondo uno studio condotto da Sara (2009), teso a dimostrare come la cultura maschilista giochi un ruolo decisivo nelle scelte compiute dalle donne del nucleo parentale, in numerose aree della Thailandia, le figlie considerate "virtuose" sono quelle che, attraverso l'attività della prostituzione, sono in grado di elevare lo status della famiglia. In altri casi, Bales (2004) ha sottolineato come, in talune provincie del nord, i due - terzi dei genitori che "vendevano" le proprie figlie per destinarle al traffico sessuale avrebbero potuto evitare di farlo, ma invece sceglievano quella soluzione per potersi permettere di comprare le televisioni a colori. Non solo, quindi, 
sfruttamento per uscire da situazioni di indebitamento e povertà, ma anche, più semplicemente, per soddisfare meri - e spesso futili - bisogni non di prima necessità. In tale situazione, dato il background culturale sopra descritto, il disvalore dell'azione di offrire la propria figlia al miglior offerente (per poi introdurla nel business della prostituzione) non è minimamente percepito. Al contrario, assume una valenza positiva, poiché permette alla figlia di assumere un ruolo nel contesto familiare.

Alla luce di quanto sopra, il coinvolgimento dei minori all'interno dell'industria del sesso in Thailandia si pone come un problema estremamente complesso, che fonda le sue radici nelle tradizionali pratiche patriarcali e trova la sua esasperazione negli effetti più drammatici della globalizzazione, quali l'estrema povertà e le migrazioni forzate ${ }^{26}$.

\subsection{La risposta normativa thailandese al traffico di persone}

Data le vicissitudini che hanno caratterizzato il traffico di persone in Thailandia - nelle sue varie forme, specialmente, per fini di sfruttamento sessuale - non deve stupire il lettore l'apprendere che la normativa a contrasto della tratta è considerata tra quelle più avanzate dei Paesi appartenenti all'ASEAN (Davy 2014: 803). Il primo intervento normativo contro tale fenomeno risale al 1928, quando il Parlamento approvò il Trafficking in Women and Girls Act, finalizzato a diminuire il numero di donne coinvolte nel business della prostituzione forzata con l'obiettivo, così, di abbattere il numero delle malattie sessualmente trasmissibili. La peculiarità di tale fonte normativa era che puniva esclusivamente l'azione di portare donne e ragazze (soprattutto di nazionalità cinese perché, ai tempi, la Thailandia era principalmente uno Stato di destinazione), dentro o fuori i confini thailandesi per fini di sfruttamento sessuale, ma la fattispecie della prostituzione forzata domestica non era individuata come tipologia di tratta. Suddetto strumento di repressione venne poi abrogato nel 1997 dal Prevention and Suppression of Trafficking in Women and Cbildren Act, il quale andò ad inasprire le pene nei confronti dei trafficanti di esseri umani e dei funzionari che, mediante corruzione, agevolavano lo svolgimento del crimine in questione. Come fa notare Obokata (2004: 50), uno degli aspetti più significativi di detto atto fu che la sua applicazione si estendeva, per la prima volta, anche ai casi in cui donne e bambini, compresi quelli delle tribù delle zone rurali, venivano trafficati internamente

\footnotetext{
${ }^{26}$ Il descritto insieme di fattori storico, culturali, economici e sociali ha condotto ad una situazione in cui, secondo il Trafficking in persons from Cambodia, Lao PDR and Myanmar to Thailand Report redatto dall'UNODC nell'agosto 2017, in Thailandia circa 140.000 persone operano nell'industria del sesso e il $90 \%$ di queste sono donne. I dati forniti dalle autorità governative thailandesi riportano che circa 77.000 persone sono coinvolte in attività di prostituzione ma, secondo le stime fornite dalle organizzazioni non governative che operano in loco, la cifra raggiunge i 300.000 individui, fra cui almeno 60.000 minori.
} 
ai confini nazionali. Tuttavia, come per la precedente misura legislativa, anche quest'ultima disciplina era principalmente orientata alla riduzione delle patologie sessualmente trasmissibili, piuttosto che ad occuparsi della tratta di persone come grave crimine contro i diritti umani delle vittime.

In questo senso, il panorama legislativo è radicalmente mutato dopo l'adozione, sul piano internazionale, del Protocollo contro la Tratta, ratificato dalla Thailandia nell'ottobre 2013. L'approccio olistico, basato sulla tutela dei diritti fondamentali dell'individuo e sul triplice sistema di prevenzione, protezione e condanna è divenuto, invero, lo scheletro su cui è stato costruito il principale strumento normativo thailandese contro il traffico di esseri umani: il Prevention and Suppression of Human Trafficking Act, B.E. 2551, approvato nel 2008 e poi emendato due volte dal Prevention and Suppression of Human Trafficking (No.2) Act, B.E. 2558 del 2015 e dal Prevention and Suppression of Human Trafficking (No. 3) Act, B.E. 2560 del 2017 (di seguito anche l'”Atto Anti-Tratta"). Seguendo lo schema dettato dal Protocollo di Palermo, si può proporre la seguente analisi.

2.3.1. Innanzitutto, l'attuale definizione di tratta si differenzia da quella adottata dal protocollo internazionale. Nonostante questa discrasia, si può apprezzare la scelta di aver utilizzato una descrizione anche più ampia del fenomeno e più aderente alla specifica situazione fattuale. E infatti, ai sensi dell'articolo 6:

Una persona che compie uno dei seguenti atti a scopo di sfruttamento è colpevole di traffico di persone:

1. procurare, acquistare, vendere, commercializzare, portare da o inviare a, detenere o confinare, ospitare o ricevere qualsiasi persona, mediante la minaccia o l'uso della forza, il rapimento, la frode, l'inganno, l'abuso di potere o la consegna di denaro o vantaggi per ottenere il consenso di una persona che ha il controllo su un'altra persona per consentire all'autore del reato di sfruttare la persona sotto il suo controllo; oppure

2. procurare, acquistare, vendere, commercializzare, portare da o inviare a, detenere o confinare, ospitare o ricevere un minore ${ }^{27}$.

Se l'atto viene interpretato ai fini dello sfruttamento, tale persona commette il reato di tratta.

Per "sfruttamento" ai sensi del paragrafo 1 si intende la ricerca di benefici dalla prostituzione, dalla produzione o distribuzione di materiale pornografico, altre forme di sfruttamento sessuale, asservimento o

${ }^{27}$ Ai sensi dell'articolo 4 dell'Atto Anti-Tratta, per "minore" si intende ogni persona al di sotto dei diciotto anni di età. 
schiavitù, lavoro o servizio forzato, costringendo un'altra persona ad essere mendicante, prelievo forzato di organi a scopo di commercio, o qualsiasi altra pratica simile che risulti da un'estorsione forzata, indipendentemente dal consenso di tale persona.

Per "lavoro o servizio forzato" ai sensi del paragrafo 2 si intende il costringere l'altra persona a lavorare o a prestare servizio con qualsiasi metodologia come segue:

1. mettendo tale persona nel timore di lesioni alla vita, al corpo, alla libertà, alla reputazione o alla proprietà, di tale persona o di un'altra persona;

2. mediante intimidazione;

3. uso della forza;

4. confisca del documento d'identità o obbligazione per debiti di tale persona o di altri; e

5. mettendo tale persona in una situazione di coercizione (traduzione mia).

Come evidenziato dal Legal Analysis of Human Trafficking in Thailand Report redatto nel 2017 dall'organizzazione non governativa Liberty Asia to Prevent Human Trafficking, la circostanza dell'abuso di potere è stata aggiunta con le più recenti modifiche, al fine di adeguare la definizione locale a quella internazionale. L'introduzione di questa specifica previsione ha permesso di includere, così, una più ampia casistica di fattori da prendere in considerazione per accertarsi se si stiano verificando fenomeni di tratta o meno, quali, a titolo esemplificativo, la situazione economica in cui versa la vittima, le caratteristiche personali, e anche la dipendenza da forme di sfruttamento familiare.

La normativa dell'Atto Anti-Tratta, poi, non si limita ad incriminare chi commette i già menzionati comportamenti, ma anche chi favorisce sotto varie forme l'esecuzione di tale reato. Come indicato dall'articolo 7:

Le persone che commettono i seguenti atti sono punite con la stessa pena del committente del reato di tratta:

(1) sostenere la commissione del reato di traffico di persone;

(2) aiutare, contribuendo con beni o procurando un luogo di incontro o un alloggio, l'autore del reato di tratta di persone; 
(3) supportare con qualsiasi mezzo l'autore del reato di tratta di persone affinché non venga arrestato;

(4) chiedere, accettare o acconsentire ad accettare una proprietà o qualsiasi altro beneficio per aiutare l'autore della tratta di persone a non essere punito;

(5) indurre, suggerire o contattare una persona a diventare membro del gruppo criminale organizzato, allo scopo di commettere il reato di tratta di persone (traduzione mia).

2.3.2. Fornita quest'estesa definizione di tratta che, nonostante taluni elementi di differenziazione, pare essere in linea con quanto stabilito dal Protocollo di Palermo, il documento in esame procede a stabilire la disciplina assistenziale prevista per le vittime, mettendo così in luce un radicale cambiamento rispetto al passato, quasi unicamente improntato sul mero regime repressivo-incriminatorio. Ai sensi dell'articolo 33, invero, il Ministry of Social Development and Human Security è tenuto a fornire una adeguata assistenza alle persone trafficate, che includa cibo, luoghi dove risiedere, trattamenti medici, riabilitazioni fisiche e psicologiche, percorsi di educazione e lavoro, supporto legale, aiuto per ritorno al paese di origine o al proprio domicilio ed infine assistenza per ottenere un risarcimento economico in sede giudiziale da parte dei trafficanti28. Come evidenziato da Roujanavong (2012: 142), queste forme di assistenza non sono soggette ad alcun limite temporale e la vittima ne può godere fino a quando non sarà tornata presso il proprio luogo di origine in condizione di sicurezza. Nel fornire aiuto, è essenziale che lo Stato prenda in considerazione le qualità più intime della persona, e dunque il sesso, l'età, l'etnia, l'appartenenza a specifici gruppi sociali e culturali, la famiglia di provenienza. Rispetto all'articolo 6 del Protocollo di Palermo, tuttavia, l'articolo 33 dell'Atto Anti-Tratta ignora di menzionare "gli speciali bisogni della vittima di traffico, specialmente dei minori" (Kranrattanasuit 2014: 122; traduzione mia) questa circostanza - che pare essere un retaggio culturale per cui il minore non vanta specifici bisogni, ma solo doveri nei confronti della famiglia - va segnalata come grave lacuna normativa, perché innalza il rischio di creare una re-vittimizzazione del bambino, alle cui necessità non viene dato il ruolo primario richiesto dallo standard internazionale.

2.3.3. Nonostante la previsione normativa regolante l'assistenza alle vittime paia predisporre un assetto conforme con i parametri internazionali (ad eccezione di quanto specificato al precedente punto), le criticità

\footnotetext{
${ }^{28}$ In questo senso, l'assistenza alle vittime è finanziata dall'Anti-Human Trafficking Fund governativo che, fra le altre spese, include anche quelle per i risarcimenti da attribuire dopo le sentenze di condanna. 
principali emergono nell'applicazione pratica: come evidenziato dal TIP Report 2020, le lacune ed inadeguatezze si riscontrano quando le autorità del Paese sono chiamate a identificare le vittime di tratta, passaggio di primaria importanza per poter poi offrire loro l'assistenza di cui all'articolo 33. Se in alcune aree della Thailandia, infatti, le forze di polizia, grazie ad un approccio multidisciplinare, sono riuscite ad ottenere significativi risultati, in altre zone si pone ancora troppa attenzione solo agli elementi più "fisici" del fenomeno, quali la detenzione forzata, il rapimento, il confinamento e l'uso dell'inganno, trascurando invece le forme alternative di coercizione, come, ad esempio, la "schiavitù per debito", che, in sempre più numerosi casi, spinge i parenti ad introdurre nell'industria della prostituzione forzata i membri più giovani del nucleo familiare.

Anche la previsione di cui all'articolo 29, in base alla quale le autorità possono tenere in custodia, in un luogo appropriato e mai in cella, una persona per 24 ore (estendibile, in virtù di provvedimento giudiziale, fino a 7 giorni) se vi sono ragionevoli motivi per credere che possa essere vittima di tratta, si pone come un limite considerevole all'attività di riconoscimento del trafficato, poiché il periodo previsto dalla normativa è spesso troppo conciso per arrivare ad una completa ed effettiva valutazione (in taluni casi, le 24 ore non sono nemmeno sufficienti a procurare alla vittima un interprete). In questo senso, l'approvazione, nel 2016, di specifiche linee guida (Guidelines to Enhance Efficiency of Human Trafficking Victim Identification) per implementare l'efficienza nel riconoscimento delle vittime ha segnato un passaggio importante nella lotta al traffico di esseri umani thailandese, ma, riportando il giudizio del TIP Report 2020, i risultati rimangono ancora ampiamente "inadeguati".

Sempre in tema di supporto vittimo-centrico, è rilevante evidenziare quanto riportato dall'articolo 36 dell'Atto Anti-Tratta:

Il funzionario competente fornisce protezione alla vittima di tratta sotto la sua tutela, indipendentemente dal luogo in cui essa soggiorna e che sia prima, durante o dopo il procedimento. Così facendo, si tiene conto anche della sicurezza dei familiari della vittima di tratta presa in considerazione.

Nel caso in cui la vittima di tratta rilasci una dichiarazione o deponga come testimone in relazione a un reato di tratta di esseri umani, la vittima, come testimone, deve essere protetta secondo le leggi relative alla protezione dei testimoni in un processo penale. 
Se una vittima di tratta deve tornare nel suo paese di residenza o di domicilio, o se i familiari della vittima di tratta vivono in un altro paese, il funzionario competente si coordina con l'agenzia governativa o privata competente in tale paese, al fine di fornire una protezione continua alla vittima di tratta e ai suoi familiari in tale paese (traduzione mia).

La citata disciplina si pone come fulcro su cui si regge l'intero apparato penalistico di repressione dell'attività criminale del traffico di persone ${ }^{29}$. Al fine di perseguire con successo i trafficanti di uomini, infatti, è essenziale che vi sia piena collaborazione della vittima, la cui testimonianza circa la propria storia di sfruttamento all'interno del processo penale assume rilevanza fondamentale per consentire la condanna del carnefice. A tal riguardo, allora, è necessario che la persona trafficata sia posta nelle condizioni, fisiche e psicologiche, per poter offrire la propria testimonianza senza ritorsioni nei suoi confronti (o nei confronti dei familiari). Va da sé che la protezione della vittima si estenda sia all'immunità rispetto a taluni reati commessi durante la vigenza del regime di tratta (e, a tal fine, è indispensabile che la persona denunci la sua condizione di vittima, pena il rischio di essere criminalizzata secondo le vigenti leggi del Paese ${ }^{30}$ ), sia al profilo della privacy, per evitare che le storie personali di chi ha sofferto forme di sfruttamento - specialmente sessuale - siano riprese e divulgate dai media locali ed internazionali, conducendo il trafficato in una sorta di re-vittimizzazione da parte dell'opinione pubblica (Roujanavong 2012: 143). Le suddette previsioni, tuttavia, talvolta sono risultate non sufficienti ad incentivare le vittime ad esporsi: come descritto dal TIP Report 2020, alcune vittime hanno continuato a riferire di essere riluttanti a partecipare ai procedimenti giudiziari a causa della paura della detenzione e dei lunghi soggiorni nei rifugi, del timore di subire ritorsioni

\footnotetext{
${ }^{29}$ Secondo le informazioni contenute nel TIP Report 2020, il governo thailandese ha riferito che, nel 2019, sono state effettuate indagini su 288 potenziali casi di traffico di persone (304 nel 2018); sono stati avviati procedimenti penali per 386 sospetti trafficanti (438 nel 2018); e sono stati condannati 304 trafficanti (316 nel 2018).

30 Ad esempio, in Thailandia è tutt'ora vigente il Prostitution Prevention and Suppression Act, B.E 2539, del 1996 che, all'articolo 4, definisce la "prostituzione" come "un rapporto sessuale, o qualsiasi altro atto, o la commissione di qualsiasi atto al fine di gratificare il desiderio sessuale di un'altra persona in modo promiscuo in cambio di denaro o di qualsiasi altro beneficio, indipendentemente dal fatto che la persona che accetta l'atto e la persona che lo commette siano delle stesso sesso o meno" (traduzione mia) e che, agli articoli 5 e 6 punisce sia "chi sollecita, segue o importuna una persona in strada, in un luogo pubblico o in qualsiasi altro luogo in modo spudorato o causa disturbo al pubblico per il fine di prostituirsi” sia "chi si unisce con un'altra persona all'interno di apposite strutture allo scopo di prostituzione" (traduzione mia). Sovente, accade che le autorità statali applichino questa disciplina piuttosto che quella delineata dall'Atto Anti-Tratta, finendo così per punire la persona trafficata con le sanzioni previste per il reato di prostituzione (Obokata 2006: 53). Come specifica Roujanavong (2012: 136), a causa delle complesse circostanze che animano la terra thailandese, gli ufficiali di polizia hanno serie difficoltà a distinguere tra $\mathrm{i}$ casi di prostituzione forzata e quelli di prostituzione volontaria. Spesso, poi, le autorità di polizia, per dimostrare l'efficienza del proprio operato nel sostenere le c.d. "leggi morali", tendono ad arrestare coloro che trovano a prostituirsi piuttosto che aprire indagini su chi costringe quelle persone a prostituirsi (Pink 2013: 166). Essere in grado di discernere correttamente fra le due fattispecie, dunque, diviene un fattore chiave per la corretta applicazione della normativa anti-sfruttamento.
} 
da parte dei trafficanti - che sovente si trovano proprio nei villaggi dove dovrebbero fare ritorno - e delle barriere linguistiche.

2.3.4. Per quanto riguarda, invece, il regime sanzionatorio da applicarsi a seguito di sentenza di condanna, l'Atto Anti-Tratta (agli articoli 52-53) ha inasprito ulteriormente le pene, andando incontro a quanto richiesto dalla comunità internazionale, soprattutto per quanto concerne la commissione dei reati nei confronti dei minori di diciotto anni (per cui è prevista anche la pena capitale) $)^{31}$.

Si noti che queste misure sono ulteriori a quelle che già sussistono all'interno del Codice penale, B.E. 2499 del 1956, che vengono parimenti utilizzate per fronteggiare le fattispecie di traffico. Ad esempio, l'articolo 282 punisce da uno a dieci anni di reclusione (che arrivano fino a venti anni, in caso di minore di quindici anni) "chi, al fine di gratificare il desiderio sessuale di un'altra persona, procura, seduce o porta via un essere umano con o senza il suo consenso per la commissione di atto indecente" (traduzione mia), mentre il successivo articolo 283 sanziona da cinque anni di reclusione fino alla pena di morte (nei casi più estremi di violenza sui minori) “chiunque, al fine di gratificare il desiderio sessuale di un'altra persona, procura, seduce o sottrae una persona per un atto indecente utilizzando mezzi ingannevoli, minaccia, atto di violenza, influenza ingiusta o coercizione con qualsiasi altro strumento" (traduzione mia). Come si può constatare, vi sono alcuni elementi di comunanza rispetto alla fattispecie di traffico per fini sessuali: in questo modo, l’imputato è accusato sia per il reato di tratta sia per il reato indicato dalle predette previsioni penalistiche, andando incontro, in caso di condanna, a lunghi periodi di regime penitenziario. Oltre a ciò, si tenga in conto che tutti i reati di cui all'Atto Anti-Tratta sono soggetti pure alla normativa dell'Anti-Money Laundering Act, B.E. 2542 (1999), in base al quale le autorità possono confiscare e congelare i ricavi provenienti dal traffico di persone, quando il trafficante viene accusato e condannato per questo reato. Tale misura è considerata, a livello mondiale, tra le più efficaci, poiché colpisce direttamente il guadagno economico, ossia la ragione principale per cui $\mathrm{i}$ trafficanti mettono in atto i comportamenti incriminati. Tuttavia, riportando l'analisi di Roujanavong (2012: 142), il governo thailandese tende a dare più importanza alla confisca dei

\footnotetext{
31 In particolare, il traffico di cui all'articolo 6 è punito, oltreché con ingenti sanzioni pecuniarie (l'equivalente di decine di migliaia di euro), con la reclusione dai quattro ai dodici anni; se la vittima è una persona dai quindici ai diciotto anni, la reclusione è da sei a quindici anni; se, invece, il reato è perpetrato contro un minore di quindici anni o contro una persona diversamente abile, è prevista la reclusione da otto a venti anni. In tutti i casi, se il trafficante provoca la morte della vittima, è punito con la pena capitale. In base al TIP Report 2020, nel 2019 i tribunali thailandesi hanno condannato circa il 74\% dei trafficanti a cinque o più anni di reclusione.
} 
beni dei trafficanti di sostanze stupefacenti piuttosto che a quelli provenienti dallo sfruttamento di persone: per questo, è necessario che le autorità competenti (magistratura e organi di polizia) siano costantemente e puntualmente istruiti sulla gravità di questo crimine e sulle multiformi sembianze che può assumere.

A completamento degli strumenti sanzionatori, ulteriori misure di repressione in tema di sfruttamento della prostituzione sono contenute anche in altre due fonti normative e, più specificatamente, nel Prevention and Suppression of Prostitution Act, B.E. 2539 (1996) e nel Cbild Protection Act, B.E. 2546 (2003). Per quanto riguarda il primo testo, si segnala che prevede una specifica previsione che interessa proprio il caso dei genitori che introducono i figli nel business della prostituzione: infatti, ai sensi dell'articolo 10, qualsiasi persona che, nella qualità di padre, madre o parente di un minore di diciotto anni, è a conoscenza della commissione del reato di prostituzione nei confronti del fanciullo e ne è connivente, è punibile con la reclusione da quattro a venti anni. Il Child Protection Act, invece, assicura la protezione delle vittime minorenni in tutti gli atti punibili da altre leggi poiché, indipendentemente dal consenso del bambino, è vietato costringere, minacciare, indurre, incoraggiare o permettere ad un minore di adottare comportamenti illeciti o che possano essere causa di reati, a prescindere dal fatto che l'intenzione sia quella di ottenere un compenso o altro.

2.3.5. Infine, sempre tenendo a riferimento la "strategia delle tre P" delineata dal Protocollo di Palermo, la Thailandia è chiamata ad attuare adeguate misure di prevenzione, ossia la via migliore per risolvere il problema del traffico di persone (Scarpa 2008). A tal riguardo, il governo thailandese ha costituito due appositi enti pubblici - denominati Committee for Prevention and Suppression of Human Trafficking e Committee for Coordinating and Monitoring the Performance in Prevention and Suppression of Human Trafficking - che, nel corso degli anni, hanno avuto il merito di porre il traffico di persone come problematica prioritaria del Paese. Da questi comitati, sono scaturite importanti iniziative tese a sensibilizzare l'opinione pubblica - inclusi i viaggiatori stranieri - sulla drammaticità del fenomeno, ad informare le potenziali vittime sulle modalità con cui agiscono i trafficanti nonché a proteggere chi, all'interno delle autorità, denuncia casi di corruzione e favoreggiamento (la tutela dei whistleblowers è stata introdotta con gli emendamenti del 2015 all'Atto Anti-Tratta). Tuttavia va segnalato che, nonostante questi interventi, le vittime, generalmente, mantengono la tendenza a dare più fiducia e credibilità ai trafficanti che alle campagne informative delle autorità, poiché, spesso, i primi hanno delle fruttuose e durature relazioni con i loro parenti, familiari o coetanei nei rispettivi villaggi di origine. Proprio grazie a questi rapporti 
fiduciari, chi traffica esseri umani si pone in una condizione privilegiata nei confronti del potenziale trafficato, che sovente si affida alla fallace prospettiva di ottenere un lavoro dignitoso e retribuito per mano del trafficante (Roujanavong 2012: 145-146).

2.3.6. A conclusione di questo paragrafo, si può constatare come la Thailandia, nel corso degli ultimi quindici anni, abbia avviato un significativo percorso - che ancora necessita di miglioramenti - per adeguare la propria normativa anti-tratta a quanto stabilito dal Protocollo di Palermo e dalle altre convenzioni internazionali a tutela dei diritti umani. Tuttavia, la risposta dell'intero sistema anti-tratta, nella sua concreta implementazione, ha dimostrato di essere carente sotto numerosi aspetti, quali, ad esempio, la corretta individuazione dei trafficanti, l'adeguato e rapido riconoscimento delle vittime di tratta da parte dei funzionari locali e lo scambio di informazioni tra le autorità del Paese (Kranrattanasuit 2014: 128). A ciò si aggiunga che, come riportato dal TIP Report 2020:

La corruzione continua a minare gli sforzi anti-tratta. Alcuni funzionari governativi sono direttamente complici dei reati di tratta, anche accettando tangenti o prestiti da proprietari di imprese e bordelli che sfruttano le vittime. Funzionari dell'immigrazione corrotti facilitano il traffico ricevendo mazzette da mediatori e contrabbandieri lungo i confini thailandesi. Rapporti attendibili indicano che alcuni funzionari corrotti proteggono i bordelli, altri luoghi di sesso commerciale, proprietari di fabbriche e di pescherecci da raid, ispezioni e procedimenti giudiziari e sono collusi con i trafficanti. Alcuni poliziotti locali, secondo quanto riferito, nascondono informazioni ai pubblici ministeri per proteggere i trafficanti. Alcuni funzionari governativi traggono profitto dalle tangenti e dal coinvolgimento diretto nell'estorsione e nello sfruttamento dei migranti (traduzione mia).

Per tutte queste ragioni, sebbene la situazione sia migliorata nel corso degli anni, ad oggi la Thailandia, secondo il giudizio annuale che viene fornito dal TIP Report, ancora non rispetta lo standard minimo richiesto per eliminare il fenomeno della tratta di persone ${ }^{32}$.

\footnotetext{
32 Per completezza, si evidenzia che il TIP Report 2020, che colloca gli Stati mondiali allinterno di una graduatoria che va da uno (equivalente al rispetto degli standard minimi per la lotta al traffico) a tre (equivalente al mancato rispetto di detti standard), posiziona la Thailandia nel tier 2, confermando la classificazione fornita anche nei precedenti due anni. In questo senso, le misure normative introdotte dal 2015 e il recente impegno profuso nella lotta al traffico le hanno permesso di uscire dal tier 3, dove si è trovata tra il 2014 ed il 2015, cioè dalla situazione in cui gli Stati Uniti, come misura sanzionatoria, cessano di fornire assistenza non umanitaria e non connessa al commercio al Paese inadempiente rispetto agli obblighi internazionali nella lotta al traffico di esseri umani.
} 
Come si è cercato di illustrare, i motivi per cui questa attività criminale - e specialmente il traffico di minori ai fini dello sfruttamento sessuale - continua ad essere presente in modo così massiccio nel Paese sono innumerevoli e risalgono nel tempo, ma ciò che preme di più evidenziare è che l'impianto normativo, così come descritto, seppure orientato verso un approccio vittimo-centrico, continuerà a trovare ostacoli nella sua implementazione se prima non viene interrotto quel meccanismo sociale che "autorizza" i genitori (o, più in generale, l'intero nucleo familiare) a consegnare i propri figli minorenni nelle mani dei trafficanti, al fine di ottenerne un beneficio di natura economica ${ }^{33}$. Poiché l'impianto normativo richiede che la vittima giochi un ruolo essenziale nel riconoscersi come tale e - dunque - nell'individuare il proprio sfruttatore al fine di implementare le adeguate misure di protezione e repressione, viene da chiedersi come questo scenario possa realizzarsi laddove il bambino non percepisce lo sfruttamento da parte dai propri parenti, ma, anzi, prova orgoglio perché sta onorando il proprio dovere familiare. Il sistema delle “tre P" funziona correttamente nel momento in cui la vittima riconosce di essere tale o viene riconosciuta come tale dalla comunità e da chi è chiamato a svolgere questo ruolo: nel caso thailandese, ciò dovrebbe comportare la rottura di uno schema culturale che, rispettando i dettami della vigente normativa anti-tratta, pone i genitori nel ruolo di trafficanti (o, comunque, di coloro che partecipano al processo del traffico) e i figli nel ruolo di vittime (inconsapevoli - ma, come si è visto, il consenso è irrilevante quando si verificano forme di sfruttamento). Le quali, a loro volta, dovrebbero essere identificate dal funzionario di polizia locale, chiamato a riconoscere come illegale la ultracentenaria pratica di consegnare i propri bambini alle varie forme di lavoro sessuale e, dunque, a vedere nel minore una vittima e non una fonte "autorizzata" di guadagno.

Va da sé che l'implementazione di questo nuovo paradigma richieda un mutamento di prospettive socioculturali di portata assoluta. Nel prossimo capitolo si forniranno alcune suggestioni per iniziare a tracciare un percorso in questa direzione.

\section{Trasformare le norme: una teoria}

L'approccio istituzionale al problema del sex trafficking in Thailandia, basato in prevalenza sugli strumenti del diritto ufficiale - imposizione di diritti “altri” o inasprimento di sanzioni -, si è rivelato, come si è detto (cfr.

${ }^{33}$ In linea con questo, si è riscontrato che i precedenti giudiziari disponibili, aventi ad oggetto questo tipo di relazioni, sono pressoché assenti. 
supra \$2), perlopiù inefficace. L'inadeguatezza del modello puramente "legalistico" è asserita esplicitamente da Montgomery, secondo cui, in Thailandia, "i tentativi di contrastare il problema della prostituzione minorile attraverso la legislazione internazionale sul traffico di persone $[\ldots]$ non hanno avuto sempre successo a livello locale [grassroots level], né sono state in grado di identificare priorità e bisogni dei minori coinvolti" (2011: 776; traduzione mia). Montgomery suggerisce, quale strategia alternativa, l'adozione di un approccio che rifletta sulle radici culturali del sex trafficking (2011: 798-9), offrendo, in tale direzione, significativi spunti di carattere etnografico (2009, 2011, 2014). L’idea - implicita in Montgomery - è che i fattori culturali, nel contesto thailandese, esercitino - più del timore di sanzioni “ufficiali" - un'influenza decisiva sulla condotta dei genitori che impiegano i propri figli nel business della prostituzione.

L'aspetto cruciale, su cui gli studi di Montgomery invitano a riflettere, è che la predisposizione di un intervento normativo efficace vòlto a modificare una condotta collettiva - uno schema di condotta, cioè, condiviso da un gruppo di individui - non possa prescindere da un'analisi delle motivazioni che muovono la collettività verso l'attuazione di quella condotta. Tale passaggio - la traduzione del dato antropologico inerente alle motivazioni in indicazioni sulle policy normative da adottare - risulta tuttavia appena accennato negli studi di Montgomery, probabilmente a causa del mancato riferimento a un apparato concettuale - a una teoria del cambiamento sociale - in tal senso convincente. Al fine di sopperire a tale carenza, illustriamo, nella presente sezione, le caratteristiche salienti di una teoria del cambiamento sociale - formulata nell'opera Norms in the Wild. How to Diagnose, Measure and Change Social Norms (2016) della filosofa e sociologa Cristina Bicchieri - che, a nostro avviso, ben si presta alla rielaborazione delle conclusioni raggiunte da Montgomery in un orientamento normativo finalizzato alla limitazione del fenomeno del sex trafficking in Thailandia (cfr. infra \4). La scelta di tale approccio metodologico, naturalmente, non implica una acritica adesione, da parte nostra, alla teoria di Bicchieri ${ }^{34}$. Intendiamo invece in questa sede, più semplicemente, dar conto di un apparato concettuale, perlopiù ignorato dalla letteratura sociologico-giuridica italiana; apparato concettuale che, pur basato su modelli e nozioni fondamentali ("norma", "aspettativa sociale", ecc.) 35 , nasce

\footnotetext{
34 Per alcuni rilievi critici sulla teoria di Bicchieri cfr. Guala (2017: 104-105, 107).

35 L'avvicinamento alla teoria di Bicchieri comporta, per il sociologo e il filosofo del diritto, un distacco dall'apparato concettuale tipico delle ontologie della norma (in ogni sua veste) cd. "classiche". Bicchieri, in altre parole, riformula il lessico della teoria delle norme modificando l'intensione tipica di concetti come "consuetudine", "aspettativa", ecc. Per questa ragione, in questa sede, ogni riferimento alla letteratura tradizionale (in ottica comparativa rispetto alla teoria di Bicchieri) rischierebbe di risultare inconferente. Cfr. anche infra.
} 
espressamente come guida "pratica" al cambiamento sociale, in senso (almeno potenzialmente) affine, come si è detto, ai suggerimenti emersi dalle ricerche sul sex trafficking in Thailandia.

\subsection{Concetti fondamentali}

La teoria di Bicchieri si propone di distinguere analiticamente i fattori che influenzano il prodursi di condotte collettive, allo scopo di identificare diverse strategie che possano indurne il cambiamento.

Riproduciamo (fig. 1) la struttura di base della condotta collettiva secondo Bicchieri. 亡̀ “condotta collettiva”, in particolare, uno schema di condotta condiviso da più individui, risultato di due diversi fattori: (i) l'esistenza di una credenza (belief); e (ii) il fatto che l'esistenza di tale credenza determini una preferenza (preference) $)^{36}$ dell'individuo, tra le varie opzioni di condotta disponibili, proprio per la condotta che ci si propone di analizzare.

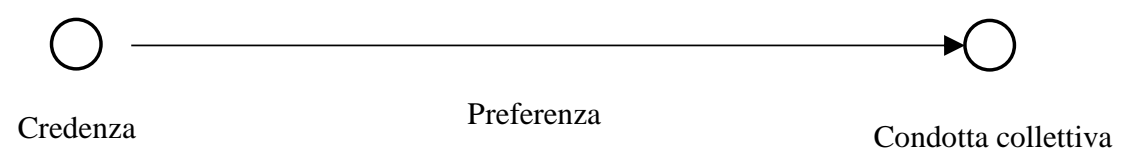

(comune a più individui)

Fig. 1 - Schema di base della condotta collettiva

Una fondamentale distinzione, tracciata da Bicchieri (2016: 2), identifica due diverse species del genus "condotta collettiva" in base al tipo di "credenza" che indirizza la preferenza: (i) se la credenza non riguarda la condotta o le credenze di altri individui, afferenti a un gruppo di riferimento (reference network) rilevante ${ }^{37}$, la condotta collettiva che ne risulta è detta indipendente (independent); (ii) se la credenza riguarda la condotta o

\footnotetext{
${ }^{36}$ Secondo il lessico concettuale di Bicchieri (2016: 6-7), la nozione (tecnica) di "preferenza" non coincide con la nozione (a-tecnica) di "gradimento": è "gradimento", infatti, soltanto uno dei fattori che, in astratto, determinano il venir in essere di una "preferenza". Ad esempio, la scelta di uno tra due gusti di gelato può risultare non soltanto da considerazioni di gradimento ("gradisco la vaniglia più del cioccolato"), ma anche da altri fattori (intolleranze, ecc.): “ciò che 'preferenza' significa davvero è che, in una determinata situazione, se io scelgo A rispetto a B vuol dire che, tutto considerato [all things considered], preferisco A" (traduzione mia; corsivo nell'originale).

${ }^{37}$ È "gruppo di riferimento rilevante", nel lessico di Bicchieri, "l'insieme di persone la cui opinione è per noi rilevante nel momento in cui dobbiamo prendere determinate decisioni" (2016: 14; traduzione mia), a prescindere dalla loro vicinanza in termini di spazio (il gruppo di riferimento può anche non essere locale: ad esempio, la famiglia di un individuo emigrato, rimasta nel Paese di origine) e di tempo (il gruppo di riferimento può anche non esistere nel tempo presente: ad esempio, gli antenati).
} 
le credenze di altri individui appartenenti al gruppo di riferimento, la condotta collettiva che ne risulta è detta interdipendente.

3.1.1. Sono condotte indipendenti le condotte determinate da credenze non-sociali: credenze che non sono, cioè, influenzate da ciò che altri individui fanno o credono. Sono condotte indipendenti, secondo Bicchieri, le condotte collettive motivate da credenze morali, dalla propria fede religiosa, o da considerazioni di carattere utilitaristico, che rispondono a necessità personali (ad esempio, aprire l'ombrello quando piove per evitare di bagnarsi).

Come si nota, nel caso delle condotte indipendenti, la "collettività" della condotta (il fatto che lo schema di comportamento sia condiviso da più individui) è un mero accidente, dovuto al fatto che più individui condividono gli stessi principi morali, la stessa fede religiosa, o le stesse necessità. La species di condotta collettiva "consuetudine sociale" è estremamente ampia e comprende sub-species tra loro profondamente diverse. In particolare, Bicchieri (2016: 12) distingue tra: (i) credenze fattuali (factual beliefs) (relative a ciò che accade), condizionate dalla necessità di rispondere a bisogni personali: ad esempio, "impiego mio figlio nel business della prostituzione poiché mi occorre denaro per sopravvivere"38; (ii) credenze normative personali (personal normative beliefs) (credenze relative a ciò che dovrebbe [should] accadere), condizionate da credenze morali/religiose personali: ad esempio, "impiego mio figlio nel business della prostituzione poiché ritengo sia giusto".

Bicchieri denomina il legame tra le due sub-species di "credenza", attraverso la mediazione della preferenza, e una determinata condotta, consuetudine sociale (social custom) ${ }^{39}$ (fig. 2) nel caso delle credenze fattuali; e norma morale/religiosa (moral/religious norm) (fig. 3) nel caso delle credenze normative personali. È dunque "consuetudine sociale" uno "schema di comportamento a cui gli individui preferiscono attenersi incondizionatamente poiché risponde alle loro necessità" (Bicchieri 2016: 15; traduzione mia); è "norma morale/religiosa" uno schema di comportamento a cui gli individui preferiscono attenersi incondizionatamente poiché corrisponde alle loro credenze morali/religiose. Avvalendosi dell'avverbio "incondizionatamente", Bicchieri si riferisce alla natura "non-condizionale" della preferenza che "media" tra

\footnotetext{
38 Significativamente, Bicchieri (2016: 41) annovera tra le credenze fattuali le credenze relative alla possibilità di incorrere in una sanzione giuridica: ad esempio, "mi astengo da impiegare mio figlio nel business della prostituzione poiché in quel caso mi aspetto di essere sanzionato".

39 Come si vedrà anche infra, il lessico concettuale adottato da Bicchieri si pone in forte discontinuità rispetto al linguaggio tradizionale della filosofia e della sociologia del diritto. È "consuetudine" [custom], ad esempio, secondo una classica definizione dell'antropologo Edward Sapir "la totalità degli schemi di comportamento supportati dalla tradizione e tipici della vita del gruppo, [concettualmente] opposti alle attività personali e casuali dell'individuo. Non si applica alle attività comuni che appaiono determinate da considerazioni di carattere biologico" (1931: 658; traduzione mia).
} 
credenza e condotta: la preferenza è "non-condizionale”, secondo il lessico concettuale di Bicchieri, poiché tale preferenza prescinde dalla conoscenza e dalla valutazione di - dalla coordinażione con - ciò che altri individui credono o del (con il) modo in cui altri individui si comportano.

3.1.2. Sono condotte interdipendenti le condotte determinate da credenze sociali: credenze, cioè, influenzate da ciò che altri individui fanno o credono. Sono dunque condotte interdipendenti, secondo Bicchieri, le condotte collettive motivate da una determinata species di "credenza", detta "aspettativa sociale" [social expectation] ${ }^{40}$. È, in particolare, "aspettativa" "una credenza relativa a ciò che accade o che dovrebbe accadere" (2016: 11; traduzione mia); l'aspettativa è, inoltre, "sociale" quando tale credenza riguarda "i comportamenti o le credenze di altri individui" (ibidem). La species "aspettative sociali" si distingue, a sua volta, in due sub-species:

1. aspettative empiriche (empirical expectations): aspettative sociali relative al modo in cui gli altri individui si comportano: ad esempio, "impiego mio figlio nel business della prostituzione poiché credo (mi aspetto) che anche gli altri lo facciano (che anche gli altri impieghino i propri figli nel business della prostituzione)";

2. aspettative normative (normative expectations): aspettative sociali relative a ciò in cui gli altri individui credono: ad esempio, "impiego mio figlio nel business della prostituzione poiché credo (mi aspetto) che gli altri credano che sia giusto farlo". Le aspettative normative sono credenze "di secondo livello" [second-order beliefs] (Bicchieri 2016: 12), in quanto identificano credenze-di (su)-credenze.

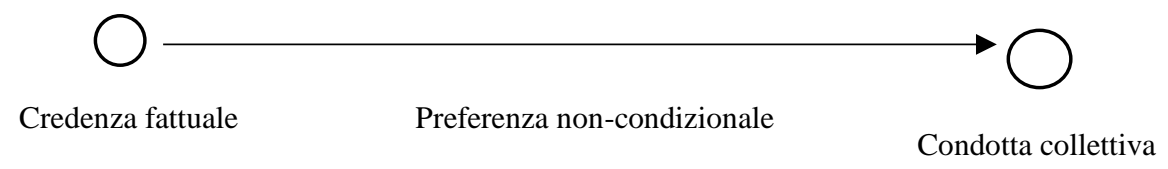

Fig. 2 - Schema della consuetudine sociale

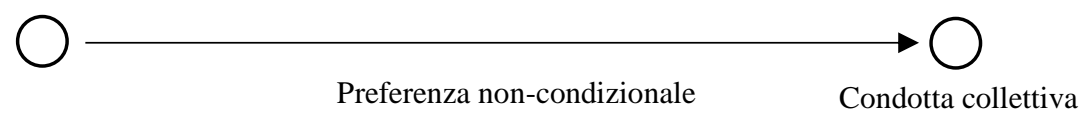

Credenza normativa personale

\footnotetext{
${ }^{40}$ La più importante anticipazione della nozione di "aspettativa" in àmbito sociologico è, come è noto, in Luhmann (cfr. almeno Luhmann 1969; 1973). Il significato con cui il termine è impiegato da Bicchieri, come già accennato, non coincide con il suo significato "classico" (in senso luhmaniano).
} 
Fig. 3 - Schema della norma morale/religiosa

Come si nota, nel caso delle condotte interdipendenti, la "collettività" della condotta non è, come nel caso delle condotte indipendenti, un mero "accidente", dovuto al fatto che più individui condividono casualmente la stessa credenza fattuale o normativa (personale); la "collettività" della condotta è invece, precisamente, il prodotto della reciproca coordinazione tra i comportamenti di più individui.

La diversa natura - empirica o normativa - dell'aspettativa che determina il prodursi di una certa condotta collettiva dà vita a due distinti tipi di relazioni, che, attraverso la mediazione della preferenza - in questo caso condizionale, poiché influenzata dalla conoscenza e dalla valutazione di ciò che altri individui credono o del modo in cui altri individui i comportano - legano aspettative e condotte:

1. nel caso delle (sole) aspettative empiriche: norma descrittiva (descriptive norm), uno "schema di comportamento a cui gli individui preferiscono attenersi a condizione che credano [believe] che anche la maggior parte delle persone nel loro gruppo di riferimento si attenga allo stesso schema" (fig. 4) (Bicchieri 2016: 19; traduzione e corsivo miei);

2. nel caso della compresenza tra aspettative empiriche e normative: norma sociale [social norm], uno schema di comportamento "a cui gli individui preferiscono attenersi a condizione che credano che (a) anche la maggior parte delle persone nel loro gruppo di riferimento si attenga allo stesso schema e che (b) la maggior parte delle persone nel loro gruppo di riferimento creda che ci si debba attenere a quel comportamento" (fig. 5) (Bicchieri 2016: 35). Nella definizione di "norma sociale", la condizione di cui alla lettera (a) identifica un'aspettativa empirica; la condizione di cui alla lettera (b) un'aspettativa normativa.

Norme descrittive e norme sociali identificano dunque, secondo Bicchieri, schemi di comportamento la cui condizione di esistenza è duplice:

1. prima condizione: l'esistenza di aspettative empiriche (norma descrittiva) e di aspettative empiriche $e$ normative (norma sociale);

2. seconda condizione: il fatto che la preferenza dellindividuo per una determinata condotta sia dovuta precisamente all'esistenza di tali aspettative empiriche e normative.

Fig. 4 - Schema della norma descrittiva 


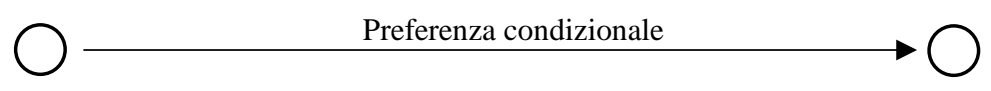

Aspettativa empirica

Condotta collettiva

Fig. 5 - Schema della norma sociale

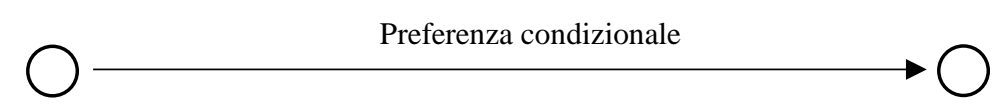

Aspettativa empirica $e$

Condotta collettiva

aspettativa normativa

La precisazione di cui al punto 2 - circa la natura della preferenza condizionale quale nesso causale tra aspettative sociali e condotta nella struttura della norma sociale - è significativa, poiché non è possibile escludere a priori che le aspettative normative coesistano con altri tipi di "credenza". Ad esempio: un individuo, pur ritenendo che i membri del suo gruppo di riferimento impieghino i propri figli nel business della prostituzione (aspettativa empirica) e che essi credano che sia opportuno farlo (aspettativa normativa), preferisce impiegare suo figlio nel business della prostituzione poiché crede che, in questo modo, rispetterà le tradizioni dei suoi antenati (credenza normativa personale). In tal caso, la relazione tra credenze e condotta si esprime in forma di norma morale/religiosa (e non di norma sociale, pur sussistendo aspettative empiriche e normative).

Raduniamo nella seguente tabella (tab. 1) i diversi tipi di relazione che legano credenze individuali e condotte collettive.

\begin{tabular}{|l|l|l|}
\hline Credenza & Preferenza & Relazione credenze-condotta \\
\hline Credenze normative personali & Non-condizionale & Norma morale/ religiosa \\
\hline Credenze fattuali & Non-condizionale & Consuetudine sociale \\
\hline Aspettative empiriche & Condizionale & Norma descrittiva \\
\hline
\end{tabular}


In via di principio, un intervento finalizzato a modificare una condotta collettiva indipendente non implica la coordinazione tra più individui. Poiché tali condotte sono motivate da credenze non-sociali, interne alla sfera individuale, l'intervento vòlto a modificarle è un intervento finalizzato a modificare convinzioni proprie di un individuo che nulla hanno a che fare con ciò in cui gli altri individui credono o con il modo in cui gli altri individui si comportano.

Le credenze normative personali rappresentano, secondo Bicchieri, "ostacoli maggiori” (2016: 6) - rispetto alle aspettative - agli interventi di cambiamento, data la (intuitiva) difficoltà nel modificare le convinzioni religiose o morali di un individuo.

Le credenze fattuali - fondate sull'identificazione di bisogni e necessità personali (cfr. supra $\ 3.1 .1$ ) possono invece, in alcuni casi, implicare un intervento coordinato. Quando, in relazione a determinate credenze fattuali, "scopriamo un alternativa, un metodo migliore per soddisfare il nostro bisogno" (2016: 16; traduzione mia) tale alternativa può risultare da uno sforzo coordinato della comunità. Ad esempio, l'abbandono della condotta "impiegare i propri figli nel business della prostituzione", se fondata su credenze fattuali, potrebbe richiedere la modifica della credenza "devo impiegare i miei figli nel business della prostituzione poiché mi occorre denaro per sopravvivere". Un individuo potrebbe infatti abbandonare tale credenza ove fosse indotto, tra le altre cose, a credere nell'esistenza di fonti di guadagno alternative (incremento dell'offerta di lavoro, implementazione di policies di sostegno economico per i meno abbienti, ecc.) la cui creazione, però, implica, appunto, uno sforzo coordinato della comunità. In questo caso (e altri simili), "la consuetudine sociale è uno schema di condotte individuali, ma modificarla implica l'introduzione di interdipendenze" (ibidem).

3.2.2. Una seconda distinzione tra species di intervento occorre tra interventi vòlti a modificare norme descrittive

e interventi vòlti a modificare norme sociali.

Secondo Bicchieri:

quando le condotte sono interdipendenti, agire da soli è rischioso [ma...] deviare rispetto a quanto prescritto da una norma sociale è normalmente più gravoso, sul piano personale e sociale, che deviare rispetto a quanto prescritto da una norma descrittiva. [... la deviazione rispetto alla condotta prescritta da una norma sociale] può far vacillare il rapporto di reciproca fiducia determinando un danno [...] a livello sociale [...]. Al contrario, violare una convenzione [un tipo di consuetudine sociale] come una regola grammaticale danneggia solo il parlante, che non sarà in grado di comunicare in maniera intelligente con altri. A meno che tale deviazione non sia un'epifania di altre e più sinistre intenzioni o mancanze, il biasimo sociale è assente (Bicchieri 2016: 108; traduzione mia). 
Sia gli interventi vòlti a modificare una norma descrittiva, sia gli interventi vòlti a modificare una norma sociale, che interessano condotte collettive interdipendenti, risultano efficaci quando il loro effetto è una diversa modalità di coordinazione tra gli individui rispetto alla modalità di coordinazione imposta dalla norma (sociale o descrittiva) che l'intervento intende modificare. Data la differenza strutturale, tra norma sociale e norma descrittiva, le modalità operative dell'intervento risultano tuttavia diverse:

[1] a coordinazione, nel caso delle norme descrittive, si può ottenere facilmente attraverso la comunicazione. La comunicazione può assumere varie forme, ma è efficace solo quando ha raggiunto il maggior numero di individui che compongono il gruppo di riferimento rilevante. In questo caso, sarà nel nostro interesse adeguarci al cambiamento, poiché non farlo ci porrebbe in una posizione svantaggiosa. [Ottenere] coordinazione, nel caso dell'abbandono di norme sociali, è molto più complesso [...]. Nel caso delle norme sociali, la sola comunicazione, in qualsiasi forma, anche se accompagnata dalla credenza comune che gli altri individui siano esposti allo stesso messaggio, di solito non è sufficiente a generare fiducia nel fatto che la norma stia effettivamente cambiando (Bicchieri 2016: 108-9; traduzione e corsivo miei).

L'intervento vòlto a modificare norme sociali risulta particolarmente complesso, poiché implica la necessità di modificare sia aspettative empiriche, sia aspettative normative (cfr. supra \$3.1.2). In questo caso, secondo Bicchieri (2016: 119-120), il perno attorno al quale il cambiamento sociale può svilupparsi è identificato dalle aspettative empiriche. Ciò è evidente, come illustrato con riferimento a diversi campi dell'agire sociale (Bicchieri and Xiao 2009), soprattutto nei casi in cui aspettative empiriche e aspettative sociali divergono: nei casi in cui, cioè, gli individui preferiscono adottare una determinata condotta, sebbene credano che tale condotta sia sconveniente e che sia condannata dai componenti del gruppo di riferimento (aspettative normative contra condotta), poiché tale condotta è estremamente diffusa e comunemente praticata (aspettative empiriche pro condotta). Le aspettative empiriche assolvono infatti a una funzione cruciale in relazione al cambiamento sociale, poiché sono in grado di “indebolire" [weaken] le aspettative normative su cui, in ultimo, si fonda la norma sociale (Bicchieri 2016: 120).

\section{Trasformare le norme in Thailandia: primi spunti di riflessione}

Avanziamo, nella presente sezione, alcune suggestioni relative al possibile contributo della teoria di 
Bicchieri all'interpretazione del fenomeno del sex trafficking in Thailandia e alla predisposizione di strumenti normativi (sociali e giuridici) finalizzati al suo contenimento.

Come si è detto (cfr. supra \3.2), secondo la teoria di Bicchieri l'elaborazione di un intervento vòlto a modificare una condotta collettiva implica un duplice sforzo preliminare:

1. l'individuazione della species di "credenza" (credenza normativa personale, credenza fattuale, aspettativa empirica, aspettativa normativa) che induce più individui a preferire una certa condotta collettiva. La domanda a cui, nel caso della Thailandia, occorre trovare risposta è dunque: In virtù di quale species di credenza gli individui preferiscono impiegare i propri figli minori nel business della prostituzione?

2. l'individuazione della species di "relazione" (norma morale/religiosa, consuetudine sociale, norma descrittiva, norma sociale) che lega la credenza (o le credenze) individuate al punto 1 a una certa condotta collettiva, attraverso la mediazione della preferenza. La domanda a cui, nel caso della Thailandia, occorre trovare risposta è dunque: Quale species di norma regola la condotta degli individui che impiegano i propri figli minori nel business della prostiturione?

La risposta alle due domande non è, come si vedrà, necessariamente univoca.

In $\iint 4.1$ e 4.2 offriamo, nel tentativo di rispondere alle predette domande, alcuni, primi, spunti di riflessione, a partire da una ricognizione della letteratura di stampo socio-antropologico sulla società thailandese. Auspichiamo, per il futuro, un'indagine più approfondita, basata su dati di campo raccolti con lo specifico intento di concettualizzare la condotta del sex trafficking nell'alveo delle categorie formulate da Bicchieri.

4.1. Non v'è in àmbito socio-antropologico, allo stato dell'arte, un numero congruo di ricognizioni sulla pratica del sex trafficking in Thailandia compatibili con il metodo di Bicchieri. In particolare, a nostra conoscenza, soltanto Montgomery pone in modo esplicito la domanda: "perché alcuni genitori agiscono in modo tale da infliggere un danno così profondo ai propri figli [impiegandoli nel business della prostituzione]?” (2014: 173; traduzione mia). La risposta è, secondo Montgomery, duplice.

In primo luogo, i genitori adottano ("preferiscono" adottare) tale condotta poiché, spesso costretti in stato di povertà, ritengono (“credono") che impiegare i propri figli nel business della prostituzione sia l'unico 
modo per guadagnare denaro (ibidem). Questa risposta sembra suggerire, adottando le categorie concettuali di Bicchieri, che i genitori agiscano in base a una credenza fattuale: la credenza secondo cui l'impiego dei propri figli nel business della prostituzione comporti un incremento della propria ricchezza. Tuttavia, tale spiegazione, che identifica l'impiego dei figli minori nel business della prostituzione in Thailandia come una consuetudine sociale, appare soltanto parziale, per almeno due ragioni:

1. come ammette la stessa Montgomery, "la povertà, da sola, non spiega perché i genitori e altri adulti non lavorino" (2014: 174). Non si comprende, cioè, perché impiegare i propri figli nel business della prostituzione identifichi, in Thailandia, l'unico modo per guadagnare denaro;

2. nulla esclude che il guadagno ottenuto attraverso l'impiego dei propri figli nel business della prostituzione sia inteso per scopi ulteriori rispetto alla mera acquisizione di ricchezza personale. Montgomery pare accennare a questa eventualità quando afferma che "i guadagni dei bambini sono spesso [...] il mezzo per mantenere integra la comunitä" (ibidem; traduzione e corsivo miei) ${ }^{42}$.

In secondo luogo, i genitori impiegano i propri figli nel business della prostituzione poiché credono che $i$ propri figli, in quanto tali, siano loro debitori (ibidem). Montgomery si riferisce, in particolare, a un fenomeno culturale chiamato in Thailandia 'bun khur(บุญคุณ), espressione che l'autrice traduce con "debito di gratitudine" [debt of gratitude] (letteralmente: “favore che determina un obbligo"). È bun khur, in particolare, il debito di gratitudine in virtù del quale i figli incorrono nei confronti dei propri genitori per il solo fatto di essere nati (Mulder 1979: 67); e in virtù del quale i figli sono obbligati a contribuire alla sopravvivenza - in termini economici - dei propri genitori (Blanc-Szanton 1985).

Al fine di determinare la natura (indipendente o interdipendente) della condotta dei genitori che - in virtù dell'esistenza di un debito di gratitudine dei propri figli nei loro confronti - impiegano i propri figli nel business della prostituzione - e di predisporre, dunque, un adeguato intervento normativo finalizzato alla trasformazione di tale condotta collettiva - occorre indagare la natura della credenza nell'esistenza del bun khur. Occorre, in particolare, comprendere se tale credenza sia riconducibile alla species "credenza normativa personale" - e, di conseguenza, se l’impiego dei figli minori nel business della prostituzione sia interpretabile

\footnotetext{
${ }^{42}$ Secondo altri (cfr. Bales 1999), la volontà di incrementare il patrimonio personale sarebbe invece mera espressione di una radicata tendenza della popolazione thailandese al consumismo.
} 
come condotta motivata da una norma religiosa/morale - oppure se tale credenza sia invece (o anche) riconducibile alle categorie "aspettativa empirica" e/o "aspettativa normativa" e, di conseguenza, a una norma empirica e/o a una norma sociale.

In tal senso, il dato antropologico è, ad oggi, piuttosto carente. Riportiamo tuttavia di seguito gli spunti più interessanti.

Il fenomeno culturale del bun khur è comunemente inteso quale prodotto della "dimensione morale [moral scale] del buddismo thailandese" (Tantiwiramanond and Pandey 1987: 34; traduzione mia) e, in quanto tale, come fattore che influenza in modo significativo la condotta dei genitori (Montgomery 2009: 136). Tuttavia, non è possibile escludere a priori che la condotta "impiegare i propri figli nel business della prostituzione" sia motivata da credenze diverse rispetto alle credenze morali di matrice buddista. In questo senso, Montgomery (2014: 177-8) documenta l'esistenza di una forte pressione sociale che, in Thailandia, colpisce i figli - e indirettamente i loro genitori - che, rifiutando di prostituirsi, gettano discredito sulla propria famiglia, che risulta "grandemente sgradita dagli altri abitanti del villaggio" (traduzione mia). A ciò che, con tutta evidenza, pare identificare un'aspettativa normativa (la credenza secondo cui gli altri abitanti del villaggio credono giusto impiegare i figli nel business della prostiturione) si affianca, secondo Montgomery, un'aspettativa empirica:

Le madri negano, in tutte le occasioni, di aver chiesto ai propri figli di diventare prostituti, ma data la diffusione di tale condotta nei quartieri bassi, quando un bambino intuisce il bisogno di guadagnare denaro non deve far altro che guardarsi intorno e osservare il modo in cui le loro sorelle, i loro fratelli e amici si comportano per capire ciò che ci si aspetta da loro (Montgomery 2014: 177; traduzione e corsivo miei).

Tale aspettativa empirica, talvolta, contrasta esplicitamente (secondo una dinamica sociale illustrata supra, $\left.\int 3.2 .2\right)$ con la circostanza che in Thailandia "la prostituzione non è mai intesa come un lavoro [...] socialmente accettabile" (Montgomery 2014: 175; traduzione mia), corrispondente all'aspettativa normativa "credo che gli altri non credano che impiegare i propri figli nel business della prostituzione sia giusto". In questo senso, dal dato antropologico traspare come i figli minori abbiano spesso una percezione distorta delle aspettative della società in relazione all'esercizio della prostituzione. Ciò avviene quando l'aspettativa normativa dei figli relativa alla credenza degli altri abitanti del villaggio circa la sconvenienza, sul piano sociale, 
dell'esercizio della prostituzione è "indebolita" da due fattori:

1. dalla già menzionata aspettativa empirica "credo che gli altri membri del villaggio impiegbino i propri figli nel business della prostituzione";

2. dall'aspettativa normativa "credo che gli altri membri del villaggio credano sia giusto impiegare i propri figli nel business della prostituzione in virtù del debito di gratitudine dei figli nei confronti dei genitori”.

La distorsione è, in particolare, il prodotto sia dell'enfasi che i genitori pongono sul dovere dei figli di adempiere al bun khur, sia della minimizzazione del disvalore sociale della prostituzione (cfr. supra, \ 2.3.7). Come ampiamente documentato, genitori e figli qualificano infatti spesso l'impiego dei figli nel business della prostituzione "come assolvimento di un dovere familiare piuttosto che come scambio sessuale" (Montgomery 2014: 177; traduzione mia) e la relazione con i clienti come "un rapporto di amicizia o addirittura una relazione amorosa piuttosto che come scambio finanziario tra prostituto[/a] e cliente" (172$3)^{43}$.

4.2. Sembra dunque profilarsi in Thailandia - in base a un dato antropologico, lo ribadiamo, incompleto - uno schema, in relazione alla condotta collettiva "impiegare i propri figli nel business della prostituzione", di questo tipo:

1. "impiegare i propri figli nel business della prostituzione" come condotta indipendente:

1.1. consuetudine sociale fondata sulla creden₹a fattuale: "impiegare i propri figli nel business della prostituzione è l'unico modo per guadagnare denaro";

1.2. norma morale/religiosa fondata sulla credenza normativa personale: "impiegare i propri figli nel business della prostituzione è giusto in quanto i figli assolvono in tal modo al proprio debito di gratitudine (bun khur) nei confronti dei genitori, come prescritto dall'etica buddista";

2. "impiegare i propri figli nel business della prostituzione" come condotta interdipendente:

2.1. norma descrittiva fondata sull'aspettativa empirica: "credo che gli altri abitanti del villaggio impieghino i propri

\footnotetext{
${ }^{43}$ La minimizzazione della natura sconveniente del lavoro di prostituto/a può essere indotta, ad esempio, chiedendo ai figli di elargire al cliente un piccolo dono in cambio del pagamento per la prestazione sessuale (Montgomery 2009: 135; Cohen (1982) parla di questo fenomeno come di "commercializzazione incompleta" (incomplete commercialization).
} 
figli nel business della prostituzione" (in possibile contrasto con l'aspettativa normativa "credo che gli altri abitanti del villaggio non credano che impiegare i propri figli nel business della prostituzione sia giusto”);

2.2. norma sociale fondata sull'aspettativa normativa: "credo che gli altri membri del villaggio credano che sia giusto impiegare i propri figli nel business della prostituzione".

Come si è detto (cfr. supra $\ 4.1$ ), le aspettative al punto 2.1 e al punto 2.2. indeboliscono l'aspettativa normativa, la cui esistenza è comunque documentata, "credo che gli altri membri del villaggio ritengano sconveniente impiegare i propri figli nel business della prostiturione".

Quale modalità di intervento vòlto a trasformare una condotta collettiva soccorre in un caso simile? Data la vaghezza e la scarsa profondità delle informazioni a disposizione, è impensabile delineare in questa sede un intervento specificamente adattato al contesto thailandese. Proponiamo però, quale impostazione per future ricerche sul tema, una riformulazione del modello operativo di "abbandono della norma" [norm abandonment elaborato da Bicchieri (2016: 179; fig. 6).

Secondo Bicchieri, il processo di abbandono di una norma si articola in sei passaggi (tre sul piano individuale, tre sul piano collettivo-coordinato):

1. sul piano individuale:

1.1. trasformazione delle credenze non-sociali. Nel caso thailandese, tale intervento sarebbe inteso a trasformare (i) la credenza fattuale "impiegare i propri figli nel business della prostituzione è l'unico modo per guadagnare denaro" e (ii) la credenza normativa personale "impiegare i propri figli nel business della prostituzione è giusto in quanto $i$ figli assolvono in tal modo al proprio debito di gratitudine (bun khur) nei confronti dei genitori, come prescritto dall'etica buddista'. Una trasformazione della credenza (i) potrebbe implicare la predisposizione di fonti di guadagno alternative rispetto all'impiego dei figli minori nel business della prostituzione (ad esempio, la predisposizione di politiche sociali finalizzate al sostentamento delle fasce più povere della popolazione). Una trasformazione della credenza (ii) potrebbe implicare la predisposizione di progetti educativi ad ampio raggio intesti a illustrare l'ambiguità, sul piano morale, educativo, ecc. dell'impiego dei propri figli nel business della prostituzione;

1.2. decisione collettiva (collective decision) di abbandonare la condotta. La trasformazione delle credenze non- 
sociali (punto 1.2) dovrebbe condurre, auspicabilmente, a una decisione collettiva-intesa come somma di decisioni individuali (decisione presa dalla maggior parte degli individui), e non come decisione comune - di abbandonare la condotta "impiegare i propri figli nel business della prostituzione";

1.3. credenza comune (common belief). La decisione collettiva (punto 1.2) dovrebbe determinare una situazione di fatto in cui la maggior parte degli individui crede che non sia conveniente (sul piano fattuale) e/ o che non sia giusto (sul piano morale/religioso) impiegare i propri figli nel business della prostituzione.

\section{Sul piano collettivo:}

2.1. azione coordinata (coordinated action). La credenza comune (di cui al punto 1.3) dovrebbe determinare una prima meccanica di coordinazione tra gli individui. Nel caso thailandese, questa fase potrebbe essere caratterizzata da un ribaltamento gerarchico tra l'aspettativa normativa "credo che gli altri membri del villaggio ritengano sconveniente impiegare i figli nel business della prostituzione" e l'aspettativa normativa "credo che gli altri membri del villaggio credano che sia giusto impiegare i figli nel business della prostituzione" (il cui rapporto attuale è discusso supra, $\ 4.1$ );

2.2. creazione di nuove aspettative empiriche. La coordinazione in nuce (punto 2.1) dovrebbe produrre una trasformazione delle aspettative empiriche. In particolare, in Thailandia, l'aspettativa empirica "credo che gli altri abitanti del villaggio impieghino i propri figli nel business della prostituzione" dovrebbe essere sostituita dall'aspettativa empirica (basata sull'osservazione della azione coordinata di cui al punto 2.1) "credo che gli altri abitanti del villaggio non impieghino i propri figli nel business della prostituzione".

2.3. abbandono delle aspettative normative. Come si è detto (cfr. supra, $\ 2.2 .2$ ), le aspettative empiriche assolvono una funzione cruciale nell’àmbito del procedimento di trasformazione della condotta collettiva. In particolare, le aspettative empiriche sono in grado di indebolire le (seppur diffuse) aspettative normative, come si è detto in relazione al rapporto odierno, in Thailandia, tra l'aspettativa normativa "credo che gli altri membri del villaggio ritengano sconveniente impiegare i figli nel business della prostituzione" e l'aspettativa empirica "credo che gli altri abitanti del villaggio impieghino i propri figli nel business della prostituzione". A seguito della creazione di nuove aspettative empiriche (punto 2.2), è dunque lecito attendersi un abbandono (o almeno un indebolimento) delle "vecchie" aspettative normative, qualora il contenuto delle seconde risultasse confliggente con il contenuto delle prime. Nel caso thailandese, il venir in essere 
dell'aspettativa empirica "credo che gli altri abitanti del villaggio non impieghino i propri figli nel business della prostituzione" dovrebbe condurre alla progressiva dissoluzione dell'aspettativa normativa "credo che gli altri membri del villaggio credano che sia giusto impiegare i figli nel business della prostituzione”.

\section{Conclusioni}

Come si è cercato di illustrare nei primi due paragrafi di questo elaborato, il sex trafficking minorile è una pratica diffusa, tollerata e, talvolta, incentivata all'interno dei confini thailandesi. Sebbene nel corso dell'ultimo decennio siano state adottate misure di contrasto al fenomeno sullo standard dettato dal Protocollo di Palermo, le criticità nel arginare questo grave crimine contro i diritti e la dignità del fanciullo si riscontrano non tanto nelle fonti legislative ad oggi vigenti (law in books) quanto nella applicazione delle disposizioni ivi contenute da parte delle autorità competenti (law in action). La lotta al traffico di persone, infatti, si poggia sui tre pilastri della penalizzazione, prevenzione ed assistenza che necessitano di una azione coordinata, senza la quale l'efficacia dell'intero schema di contrasto rischia di essere vanificata. Nel caso thailandese, da questo punto di vista emblematico, l'implementazione dei tre profili è, come si è detto, seriamente messa in discussione dalle radicate tradizioni del Paese e dalla sua consolidata storia di pratiche legate alla schiavitù (con particolare riferimento al caso dello sfruttamento sessuale dei minori). Ci si trova, dunque, dinnanzi ad una situazione che rovescia integralmente il sistema dei valori sanciti dal diritto internazionale dei diritti umani: in Thailandia, il disvalore si concretizza quando tale pratica non viene messa in atto. Disvalore le cui conseguenze nefaste si ripercuotono proprio sul bambino, etichettato come soggetto deviante del nucleo familiare e, più in generale, della comunità a cui appartiene.

Muovendo da tali constatazioni, esplicitate nella prima parte dell'articolo, abbiamo concisamente descritto un approccio sociologico-giuridico in grado, a nostro avviso, di fornire interessanti indicazioni, almeno in potenza, per l'elaborazione di una policy normativa finalizzata (anche) allo sradicamento del fenomeno del sex trafficking minorile in Thailandia. Tale approccio, la teoria del cambiamento sociale di Cristina Bicchieri, attribuisce una fondamentale rilevanza alle credenze (individuali e collettive) che influenzano il prodursi di condotte collettive; e individua quattro tipi di relazione tra credenze e condotte: norma morale/religiosa, consuetudine sociale, norma descrittiva, norma sociale. Ciascuna delle quattro 
"norme", secondo Bicchieri, implica, per essere modificata, l'impiego di tecniche socio-giuridiche significativamente diverse. L'applicazione di tale modello al caso thailandese, pertanto, presuppone anzitutto la corretta identificazione delle dinamiche sociali che determinano la diffusione (e la tolleranza nei riguardi) della pratica dello sfruttamento sessuale dei minori. Senza pretesa di esaustività, ma al solo fine di mostrare come la (tuttavia insufficiente) letteratura etnografica attualmente disponibile ("filtrata" dall'apparato concettuale di Bicchieri) possa fornire un contributo in tal senso, abbiamo avanzato la tesi secondo cui il sex trafficking dei minori in Thailandia risulti da un'intersezione tra consuetudine sociale (determinata dalla credenza dei genitori secondo cui l'impiego dei propri figli nel business della prostituzione comporti un aumento della propria ricchezza), norma morale (determinata dalla credenza secondo cui i figli assolvono in tal modo al proprio debito di gratitudine nei confronti dei genitori, come prescritto dall'etica buddista), norma descrittiva (determinata dalla credenza secondo cui gli altri abitanti del villaggio impieghino i propri figli nel business della prostituzione) e norma sociale (determinata dalla credenza secondo cui gli altri membri del villaggio credano che sia giusto impiegare i propri figli nel business della prostituzione). Sulla base di tali conclusioni, abbiamo descritto, nella sezione conclusiva dell'articolo, alcune linee fondamentali di un possibile intervento finalizzato alla trasformazione delle credenze poc'anzi menzionate e (da ultimo) all'abbandono della condotta collettiva identificata dalla pratica del sex trafficking. I diversi punti che articolano la nostra esposizione non devono essere, naturalmente, intesi come indicazioni definitive (poiché basati, come si è detto, su spunti etnografici perlopiù vaghi); piuttosto, come spunto di riflessione che orienti le future ricerche e riflessioni sul tema.

\section{Riferimenti bibliografici}

Bales, K. (1999), 'What Predicts Human Trafficking' https://www.freetheslaves.net/wpcontent/uploads/2015/03/WhatPredictsHumanTrafficking.pdf (ultimo accesso: 5 maggio 2021)

Bales, K. (2004), Disposable People. New Slavery in the Global Economy (Oakland: California Press)

Bicchieri, C. (2016), Norms in the Wild. How to Diagnose, Measure and Change Social Norms (New York: Oxford University Press)

Bicchieri, C. and Xiao, E. (2009), 'Do the Right Thing: But Only If Others Do So', 22(2) Journal of Behavioral Decision Making 191-208. DOI: 10.1002/bdm.621 
Blanc-Szanton, C. (1985). 'Gender and Inter-Generational Resource Allocations: Thai and Sino-Thai Households in Central Thailand'. In L. Dube and R. Parliwala (ed.), Structures and Strategies: Women, Work and Family in Asia (New Delhi: Sage) 79-102

Chuang, J. (2006), 'The United States as Global Sheriff: Using Unilateral Sanctions to Combat Human Trafficking', 27 Michigan Journal of International Law 437-494

Cohen, E. (1982), 'Thai Girls and Farang Men. The Edge of Ambiguity', 9(3) Annals of Tourism Research $403-42$

Davy, D. (2014), 'Understanding the Complexities of Responding to Child Sex Trafficking in Thailand and Cambodia', 34 International Journal of Sociology and Social Policy 793-816. DOI: 10.1108/IJSSP-10-20130103

Gallagher, A. (2001), 'Human Rights and the New UN Protocols on Trafficking and Migrant Smuggling A Preliminary Analysis', 23 Human Rights Quarterly 975-1004

Guala, F. (2017), 'Review of Cristina Bicchieri's Norms in the Wild', 10 (1) Erasmus Journal for Philosophy and Economics 101-111. DOI: 10.23941/ejpe.v10i1.282

Hitchcock, M. (2008), Tourism in Southeast Asia: Challenges and New Directions (Copenhagen: NIAS Press)

Jones, S., King, J. and Edwards, N. (2017), 'Human-Trafficking Prevention is not "Sexy": Impact of the Rescue Industry on Thailand NGO Programs and the Need for a Human Rights Approach', Journal of Human Trafficking 1-36. DOI: 10.1080/23322705.2017.1355161

Kranrattanasuit, N. (2014), ASEAN and Human Trafficking Case Studies of Cambodia, Thailand and Vietnam (Leiden: Brill)

Luhmann, N. (1969), Normen in soziologischer Perspektiver', 20 (1) Soziale Welt 28-48

Luhmann, N. (1973), Rechtsoziologie (2 voll. Reinbech: Rowohlt) 
Marshall, P. (2001), 'Globalization, Migration and Trafficking: Some Thoughts from the South-East Asian Region', occasional paper, UN Inter-Agency Project on Trafficking in Women and Children in the Mekong Sub-region

Mensendiek, M. (1997), 'Woman, Migration, and Prostitution in Thailand', 40(2) International Social Work $163-176$

Montgomery, H. (2009), 'Are Child Prostitutes Child Workers? A Case Study', 29 (3-4) International Journal of Sociology and Social Policy 130-140. DOI: 10.1108/01443330910947507

Montgomery, H. (2011), 'Defining Child Trafficking \& Child Prostitution: The Case of Thailand' 9 (2) Seattle Journal for Social Justice 775-811

Montgomery, H. (2014), 'Child Prostitution as Filial Duty? The Morality of Child-Rearing in a Slum Community in Thailand' 43 (2) Journal of Moral Education 169-182. DOI: 10.1080/03057240.2014.893420

Mulder, N. (1979), Inside Thai society. An Interpretation of Everyday Life (Bangkok: Duang Kamol)

Obokata, T. (2006), Trafficking of Human Beings from a Human Rights Perspective: Towards a Holistic Approach (Leiden: Koninklijke Brill NV)

Pink, R.M. (2013), 'Child Trafficking in Thailand: Prevention and Prosecution Challenges' 40 Asian Affairs: An American Review 163-174

Quiroz Vitale, M.A. (2018), Diritti umani e cultura giuridica: il principio di autodeterminazione e l'invenzione delle nuove schiavitù in Europa (Milano-Udine: Mimesis)

Rancati, D. (2019), 'Les effets sociojuridiques de la globalisation sur le trafic humain et sur la protection des droits humains', in M. A. Quiroz Vitale et al. (eds), Expériences juridiques sur les droits humains (Torino: L'Harmattan Italia) 76-97

Rande Taylor, L. (2003), Globalization, Parental Decision Making and Child Welfare in Rural Northern Thailand (Washington DC: University of Washington)

Riback, M. (2018), 'Uncertain Outcomes: Evaluating the Effects of the Trafficking in Persons Reports in South Africa and Thailand', 6(1) Global Societies Journal 41-68 
Roujanavong, W. (2012), 'Human Trafficking: A Challenge to Thailand and the World Community', 87 UNAFEI Resource Material 134-149

Sapir, E. (1931), 'Custom', in E. Seligman (ed.), Encyclopaedia of the Social Sciences (New York: Macmillan) 658-662

Sara, K. (2009), Sex Trafficking: Inside the Business of Modern Slavery (West Sussex: Columbia University Press)

Scarpa, S. (2008), Trafficking in Human Beings: Modern Slavery (New York: Oxford University Press Inc.)

Shelley, L. (2010), Human Trafficking: A Global Perspective (Cambridge: Cambridge University Press)

Spires, R.W. (2015), Preventing Human Trafficking: Education and NGOs in Thailand (Burlington: Ashgate

Publishing Company)

Stukas, A., Marques M. and Honeyman K. (2016), 'Human Trafficking: Factors that Influence Willingness to Combat the Issue', 46 Journal of Applied Social Psychology 529-543. DOI: 10.1111/jasp.12381

Tantiwiramanond, D. and Pandey, S. (1987), 'The Status and Role of Women in the Pre-modern Period: A Historical and Cultural Perspective', 2(1) Sojourn 125-147

Turner, B.S. (2006), Vulnerability and Human Rights (Pennsylvania: The Pennsylvania State University Press)

Wylie, G. and McRedmond, P. (2010), Human Trafficking in Europe: Character, Causes and Consequences (London: Palgrave Macmillan)

Report

International Labour Office, 2017 Global Estimates of Modern Slavery Report

Liberty Asia to Prevent Human Trafficking, 2017 Legal Analysis of Human Trafficking in Thailand Report

United Nations Office on Drugs and Crime, 2017 Trafficking in persons from Cambodia, Lao PDR and Myanmar to Thailand Report

United Nations Office on Drugs and Crime, 2020 Global Trafficking in Persons Report 


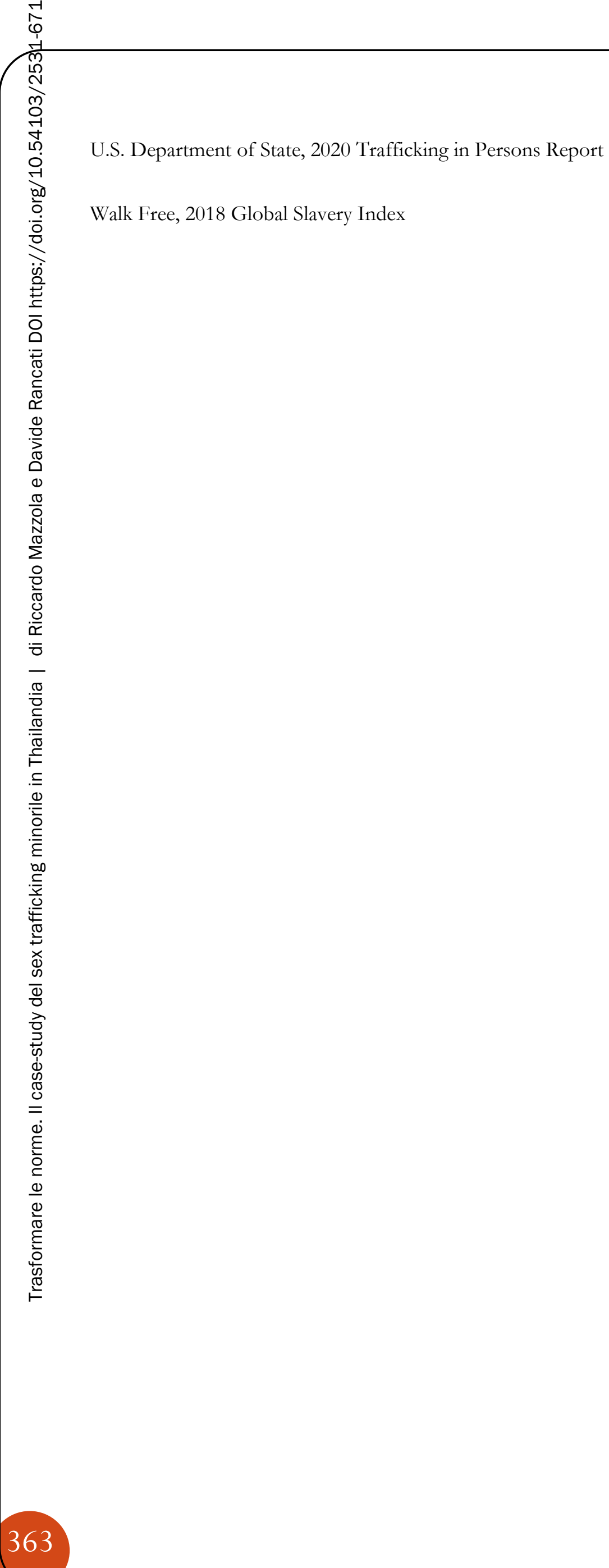

